\title{
Volume-limited radio survey of ultracool dwarfs
}

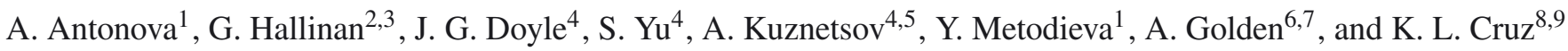 \\ ${ }^{1}$ Department of Astronomy, St. Kliment Ohridski University of Sofia, 5 James Bourchier Blvd., 1164 Sofia, Bulgaria \\ e-mail: tony@phys.uni-sofia.bg \\ 2 National Radio Astronomy Observatory, 520 Edgemont Road, VA 22903 Charlottesville, USA \\ 3 Department of Astronomy, University of California, CA 94720 Berkeley, USA \\ 4 Armagh Observatory, College Hill, BT61 9DG Armagh, N. Ireland \\ 5 Institute of Solar-Terrestrial Physics, 664033 Irkutsk, Russia \\ 6 Centre for Astronomy, National University of Ireland, Galway, Ireland \\ 7 Price Center, Albert Einstein College of Medicine, Yeshiva University, Bronx, 10461 New York, USA \\ 8 Department of Physics and Astronomy, Hunter College, City University of New York, 10065 New York, NY, USA \\ 9 Department of Astrophysics, American Museum of Natural History, 10024 New York, NY, USA
}

Received 5 December 2011 / Accepted 19 November 2012

\begin{abstract}
Aims. We aim to increase the sample of ultracool dwarfs studied in the radio domain to allow a more statistically significant understanding of the physical conditions associated with these magnetically active objects.

Methods. We conducted a volume-limited survey at $4.9 \mathrm{GHz}$ of 32 nearby ultracool dwarfs with spectral types covering the range M7-T8. A statistical analysis was performed on the combined data from the present survey and previous radio observations of ultracool dwarfs.

Results. Whilst no radio emission was detected from any of the targets, significant upper limits were placed on the radio luminosities that are below the luminosities of previously detected ultracool dwarfs. Combining our results with those from the literature gives a detection rate for dwarfs in the spectral range M7-L3.5 of 9\%. In comparison, only one dwarf later than L3.5 is detected in 53 observations. We report the observed detection rate as a function of spectral type and the number distribution of the dwarfs as a function of spectral type and rotation velocity.

Conclusions. The radio observations to date point to a drop in the detection rate toward the ultracool dwarfs. However, the emission levels of detected ultracool dwarfs are comparable to those of earlier type active $\mathrm{M}$ dwarfs, which may imply that a mildly relativistic electron beam or a strong magnetic field can exist in ultracool dwarfs. Fast rotation may be a sufficient condition to produce magnetic fields strengths of several hundred Gauss to several kilo Gauss, as suggested by the data for the active ultracool dwarfs with known rotation rates. A possible reason for the non-detection of radio emission from some dwarfs is that maybe the centrifugal acceleration mechanism in these dwarfs is weak (due to a low rotation rate) and thus cannot provide the necessary density and/or energy of accelerated electrons. An alternative explanation could be long-term variability, as is the case for several ultracool dwarfs whose radio emission varies considerably over long periods with emission levels dropping below the detection limit in some instances.
\end{abstract}

Key words. stars: low-mass - brown dwarfs - radiation mechanisms: general - radio continuum: stars - masers

\section{Introduction}

In the past few years, a number of very low mass stars and brown dwarfs (collectively termed ultracool dwarfs) have been confirmed as a new class of radio active objects. A surprising feature of these observations is the detection of periodic pulses of $100 \%$ circularly polarized emission (Hallinan et al. 2006, 2007, 2008; Berger et al. 2009; Doyle et al. 2010; McLean et al. 2011). These periodic pulses have been confirmed to be produced by the electron cyclotron maser (ECM) instability, the same mechanism that is known to produce the planetary radio emission at $\mathrm{kHz}$ and $\mathrm{MHz}$ frequencies (Treumann 2006), but it requires much more powerful kilogauss magnetic fields. Unpolarized and seemingly quiescent radio emission was present in the observations of all detected dwarfs and has alternatively been attributed to gyrosynchrotron emission and the ECM emission (see the above references).

Although much progress has been made in understanding the nature of the pulsed radio emission from these dwarfs by using its diagnostic potential, it remains unclear which characteristics distinguish radio "active" from radio "inactive" dwarfs. Possible physical characteristics include mass, temperature, activity, and rotation rate. Intriguingly, all active dwarfs have been found to have high $v \sin i$ values. This suggests two possibilities, a dependence on rotation velocity, or a dependence on inclination angle. The former suggests that slower rotators have weaker dynamo action and hence weaker magnetic fields of insufficient strength to produce detectable radio emission at the required frequency. The second scenario implies a dependence on inclination angle, i.e., a geometrical selection effect is associated with the highly beamed radio emission. A recent case study of three pulsing ultracool dwarfs does indeed confirm that all three have very high values of inclination (greater than 65 degrees). However, all three dwarfs are also confirmed to be very rapid rotators with periods of rotation $\leq 3 \mathrm{~h}$ (Hallinan et al. 2008).

Clarifying the relationship between $v \sin i$ and radio activity is imperative. If a dependence on rapid rotation underlies the observed correlation between radio luminosity and $v \sin i$, this implies that the rotation-activity relationship, which is wellestablished for main-sequence stars, extends into the substellar 
regime. If, on the other hand, the correlation between radio luminosity and $v \sin i$ indicates a geometrical selection effect, this implies that very strong magnetic fields $(\mathrm{kG})$ are ubiquitous in the substellar regime, independent of rotation rate. Thus, to clarify the relationship between $v \sin i$ and radio activity, a larger more statistically significant sample of pulsing dwarfs must be established.

The ECM emission has proved a vital diagnostic tool for remote sensing of the magnetic field strength and topologies of ultracool dwarfs. The ECM emission is generated at the electron cyclotron frequency denoted by $v_{\mathrm{c}} \approx 2.8 \times 10^{6} \mathrm{~B} \mathrm{~Hz}$ (if the electrons are not relativistic), enabling measurement of the magnetic field strength in the source region of the pulsed emission. This led to the realization of $\mathrm{kG}$ magnetic fields in late-M dwarfs (Hallinan et al. 2007) and subsequently the first confirmation of $\mathrm{kG}$ magnetic fields for an L dwarf, the latter establishing strong magnetic dynamo action out to spectral type L3.5 (Hallinan et al. 2008).

Very recently, Route \& Wolszczan (2012) reported the first detection of radio emission from a dwarf of spectral type later than L3.5 - the T6.5 brown dwarf 2MASS J1047539+212423. They have detected circularly polarized bursts at $4.75 \mathrm{GHz}$ with the Arecibo telescope and invoked the ECM mechanism as the most likely source of the emission. This latest observation confirms that detecting ECM emission remains the most promising method to measure magnetic field strengths in cooler late-type $\mathrm{L}$ and T dwarfs. Reiners \& Basri (2007) measured the magnetic field strengths of a number of late-M dwarfs through the measurement of the Zeeman broadening of the magnetically sensitive Wing-Ford FeH band. However, this technique encounters difficulties when applied to $\mathrm{L}$ and $\mathrm{T}$ dwarfs due to the heavy saturation of the $\mathrm{FeH}$ lines. Therefore, the continued search for radio emission from late- $\mathrm{L}$ and $\mathrm{T}$ dwarfs is essential to the efforts to diagnose the strength of magnetic fields in these objects.

To address the above questions we conducted a volumelimited survey of 32 ultracool dwarfs of spectral types M7 to T8.

\section{Observations and data reduction}

The target list was compiled using the 2MU2 20 pc volume limited catalog of Cruz et al. (2007). We selected field dwarfs that had no previous observations at radio frequencies and whose declination is $>-30^{\circ}$ (the latter ensuring that the observing time is sufficiently long for achieving the goals described below). This yielded 14 dwarfs in the spectral range M7-L3.5, with a view to expanding the current sample of detected pulsating ultracool dwarfs. We also selected 18 dwarfs of types L4 to T8 with the aim to detect radio emission from a source later than L4. Their properties are listed in Table 1.

The observations were conducted with the NRAO Very Large Array ${ }^{1}$ in the period 09 August-09 September 2009 using the standard continuum mode with $2 \times 50 \mathrm{MHz}$ contiguous bands. The observing frequency was chosen to be $4.9 \mathrm{GHz}$, because any ultracool dwarf observed at both $4.9 \mathrm{GHz}$ and $8.5 \mathrm{GHz}$ has been found to produce quiescent emission at $4.9 \mathrm{GHz}$ of the same luminosity or higher than that detected at $8.5 \mathrm{GHz}$. Furthermore, in the case of the ECM emission, an upper cutoff frequency is expected for the radio emission that is dependent on the maximum magnetic field of the dwarf. A survey at $4.9 \mathrm{GHz}$ thus allows for a lower magnetic field strength, which

\footnotetext{
1 The National Radio Astronomy Observatory is a facility of the National Science Foundation operated under cooperative agreement by Associated Universities, Inc.
}

additionally increases the chance of detection. For each source, a suitable phase calibrator was chosen from the VLA calibrator manual while the flux density scale was determined using the calibrators 3C 48, 3C 138, and 3C 286.

We observed each target in the survey for a duration determined by its distance, thereby ensuring a common upper limit on the luminosity of the radio emission from each dwarf. For the dwarfs spanning the spectral range M7-L3.5, the duration was chosen to match the one required to place an upper limit on the radio luminosity of $\sim 10^{13} \mathrm{erg} \mathrm{s}^{-1} \mathrm{~Hz}^{-1}$, i.e., lower than each of the previously detected pulsing ultracool dwarfs. Considering that no detection has previously been reported for late $\mathrm{L}$ and $\mathrm{T}$ dwarfs, we chose to observe each of the later type $(\geq \mathrm{L} 4)$ dwarfs for the duration required to place an upper limit on radio luminosity of $\sim 5 \times 10^{12} \mathrm{erg} \mathrm{s}^{-1} \mathrm{~Hz}^{-1}$, i.e., to partially allow for any drop off in emission that might occur with later spectral type. All targets were observed in a dynamic scheduling mode with a time resolution of $3.3 \mathrm{~s}$. This mode was therefore sufficient to detect short radio bursts, in addition to any quiescent emission.

Data reduction was carried out with the Astronomical Image Processing System (AIPS) software package using standard routines. The visibility data were inspected for quality both before and after the calibration procedures, and noisy points were removed. To make the maps we used the task IMAGR and then CLEANed the region around each source in the individual fields.

\section{Results}

We found no sources at (or near) the positions of the studied dwarfs in the respective maps, i.e., we did not detect radio emission from any of the observed dwarfs. As discussed in Sect. 2, in the case of non-detections we aimed to obtain data with a sensitivity that would allow us to place upper limits of $L_{v} \leq 10^{13} \mathrm{erg} \mathrm{s}^{-1} \mathrm{~Hz}^{-1}$ for the dwarfs in the range M7-L3.5 and $L_{v} \leq 5 \times 10^{12} \mathrm{erg} \mathrm{s}^{-1} \mathrm{~Hz}^{-1}$ for the later type dwarfs (L4-T8). We find that for all dwarfs in the sample whose distances are within $\sim 12 \mathrm{pc}$, the detection limits are at or below these values. For the most distant dwarfs, however, the limits were not reached due to insufficient sensitivity. The $3 \sigma$ upper limits on the flux and the respective radio luminosity limits are shown in Table 1.

We plot our results together with those from previous studies of UCDs at $4.9 \mathrm{GHz}$ in Fig. 1. This work increases the number of studied objects by more than $150 \%$ and lowers the upper limits on the radio luminosity. There is only one upper limit whose value is above the value of detected emission for a given spectral type - that for the M8.5 dwarf LSR J1826+3014, which is one of the most distant dwarfs in the survey $(d=13.9 \mathrm{pc})$.

McLean et al. (2012) recently reported on a $8.5 \mathrm{GHz}$ radio survey of $100 \mathrm{M}$ and L dwarfs spanning the range M4 to L4 (76 of which are ultracool dwarfs) and the detection of three new radio-emitting late-type dwarfs. Comparing the target list of their observation to ours, we found seven dwarfs in common, none of which was detected at the higher frequency either. We list these dwarfs and their flux and luminosity upper limits in the respective frequencies in Table 2 . We have calculated the upper limit on the $8.5 \mathrm{GHz}$ radio luminosity using the distances listed in Table 2 and the fluxes reported by McLean et al. (2012). Despite the small differences in the $3 \sigma$ limits on the radio flux between the two surveys, we find that the limits in radio luminosities agree well.

In addition, McLean et al. (2012) compiled a list of all previous radio observations of late-type dwarfs. Combining their 
Table 1. Sample of 32 dwarfs, their properties, and results from the present survey.

\begin{tabular}{|c|c|c|c|c|c|c|c|c|}
\hline Name & Other name & Sp. T. & $\begin{array}{c}d \\
(\mathrm{pc})\end{array}$ & $\begin{array}{c}v \sin i \\
\left(\mathrm{~km} \mathrm{~s}^{-1}\right)\end{array}$ & $\begin{array}{l}L_{\text {bol }} \\
\left(L_{\odot}\right)\end{array}$ & $\left(L_{\mathrm{H} \alpha} / L_{\mathrm{bol}}\right)$ & $\begin{array}{l}F_{(4.9 \mathrm{GHz})} \\
\left(10^{-5} \mathrm{Jy}\right)\end{array}$ & $\begin{array}{c}L_{\nu, 4.9} \\
\left(\mathrm{erg} \mathrm{s}^{-1} \mathrm{~Hz}^{-1}\right)\end{array}$ \\
\hline 2MASS J10481258-1120082 & GJ 3622 & M7 & 4.5 & 3.0 & -3.16 & -4.63 & $<6.3$ & $<1.53 \times 10^{12}$ \\
\hline 2MASS J17571539+7042011 & LP 44-162 & M7.5 & 12.5 & 33 & -3.48 & -5.01 & $<8.1$ & $<1.51 \times 10^{13}$ \\
\hline 2MASS J11554286-2224586 & LP $851-346$ & M7.5 & 9.7 & 33 & - & -4.58 & $<6.6$ & $<7.43 \times 10^{12}$ \\
\hline 2MASS J05395200-0059019 & SDSS J053951.99-005902.0 & M7.5 & 3.84 & - & - & - & $<6.0$ & $<1.06 \times 10^{12}$ \\
\hline 2MASS J12505265-2121136 & DENIS-P J125052.6-212113 & M7.5 & 11.1 & - & - & - & $<6.6$ & $<9.73 \times 10^{12}$ \\
\hline 2MASS J04351455-1414468 & - & M8 & 14 & - & - & - & $<6.0$ & $<1.41 \times 10^{13}$ \\
\hline 2MASS J02150802-3040011 & LHS 1367, LP885-35 & M8 & 12.37 & - & -3.55 & - & $<7.5$ & $<1.37 \times 10^{13}$ \\
\hline 2MASS J05392474+4038437 & LSR J0539+4038 & M8 & 10 & - & - & - & $<6.3$ & $<7.54 \times 10^{12}$ \\
\hline 2MASS J18261131+3014201 & LSR J1826+3014 & M8.5 & 13.9 & - & - & - & $<8.7$ & $<2.01 \times 10^{13}$ \\
\hline 2MASS J14284323+3310391 & GJ 3849, LHS 2924 & M9 & 11.8 & 10 & -3.62 & -4.7 & $<6.3$ & $<1.04 \times 10^{13}$ \\
\hline 2MASS J17312974+2721233 & LSPM J1731+2721 & L0 & 11.8 & 15 & -3.74 & -4.6 & $<5.7$ & $<9.50 \times 10^{12}$ \\
\hline 2MASS J09211410-2104446 & DENIS-P J092114.1-210445 & $\mathrm{L} 2$ & 12 & 15 & -4.01 & $<-6.42$ & $<7.2$ & $<1.14 \times 10^{13}$ \\
\hline 2MASS J08283419-1309198 & DENIS-P J082834.3-130919 & $\mathrm{L} 2$ & 11.6 & 33 & - & -5.68 & $<6.3$ & $<1.01 \times 10^{13}$ \\
\hline 2MASSI J0700366+315726 & - & L3.5+L6 & 12.2 & 41 & -3.96 & - & $<4.2$ & $<8.01 \times 10^{12}$ \\
\hline 2MASS J05002100+0330501 & - & L4 & 13.03 & - & -4.26 & - & $<5.1$ & $<1.04 \times 10^{13}$ \\
\hline 2MASS J04351455-1414468 & - & L4.5 & 9.8 & - & -4.12 & - & $<4.2$ & $<4.83 \times 10^{12}$ \\
\hline 2MASS J03552337+1133437 & - & L5 & 12.6 & 10 & -4.03 & - & $<4.5$ & $<8.55 \times 10^{12}$ \\
\hline 2MASS J05395200-0059019 & SDSS J053951.99-005902.0 & L5 & 13.1 & 34 & -4.2 & - & $<4.8$ & $<9.86 \times 10^{12}$ \\
\hline 2MASS J17502484-0016151 & - & L5.5 & 8 & - & - & - & $<4.3$ & $<4.82 \times 10^{12}$ \\
\hline 2MASS J21481633+4003594 & - & L6.5 & 7.9 & - & -4.07 & - & $<6.3$ & $<4.70 \times 10^{12}$ \\
\hline 2MASS J09083803+5032088 & - & L7 & 15.9 & 31 & - & - & $<11.1$ & $<3.36 \times 10^{13}$ \\
\hline 2MASS J08575849+5708514 & SDSS J085758.45+570851.4 & L8 & 11 & - & - & - & $<5.1$ & $<7.38 \times 10^{12}$ \\
\hline 2MASS J02572581-3105523 & DENIS-P J025725.7-310552 & L8 & 9.6 & - & -4.82 & - & $<9.3$ & $<1.03 \times 10^{13}$ \\
\hline 2MASS J08300825+4828482 & SDSS J083008.12+482847.4 & L9 & 13.0 & - & -4.58 & - & $<8.7$ & $<1.78 \times 10^{13}$ \\
\hline 2MASS J01365662+0933473 & SIMP J013656.5+093347.3 & $\mathrm{T} 2.5$ & $\begin{array}{c}6.4 \\
8.27\end{array}$ & - & - & - & $<7.5$ & $\begin{array}{l}<3.68 \times 10^{12} \\
<6.14 \times 10^{13}\end{array}$ \\
\hline 2MASS J15031961+2525196 & - & T5.5 & 7 & 36 & - & - & $<9.0$ & $<5.28 \times 10^{12}$ \\
\hline 2MASS J09373487+2931409 & - & T6 & 6.14 & - & -5.28 & - & $<6.6$ & $<2.98 \times 10^{12}$ \\
\hline 2MASS J21543318+5942187 & - & T6 & 18.8 & - & - & - & $<6.0$ & $<2.54 \times 10^{13}$ \\
\hline 2MASS J07271824+1710012 & - & $\mathrm{T} 7$ & 9.09 & - & -5.26 & $<-5.4$ & $<5.4$ & $<5.34 \times 10^{12}$ \\
\hline 2MASS J11145133-2618235 & - & T7.5 & $\begin{array}{c}7 \\
10\end{array}$ & - & - & - & $<6$ & $\begin{array}{l}<3.52 \times 10^{12} \\
<7.18 \times 10^{12}\end{array}$ \\
\hline 2MASS J09393548-2448279 & - & $\mathrm{T} 8$ & $\begin{array}{c}8.7 \\
5.34\end{array}$ & - & -5.69 & - & $<5.4$ & $\begin{array}{l}<4.89 \times 10^{12} \\
<1.84 \times 10^{12}\end{array}$ \\
\hline 2MASS J14571496-2121477 & GL570D & T8 & 5.88 & 30 & -5.52 & - & $<5.4$ & $<2.23 \times 10^{12}$ \\
\hline
\end{tabular}

Notes. The columns are (left to right): name of the object; other name; spectral type; distance; projected rotational velocity; bolometric luminosity; $\mathrm{H} \alpha$ luminosity; radio flux at $4.9 \mathrm{GHz}$; radio luminosity at $4.9 \mathrm{GHz}$.

References. Spectral types, distances and $v \sin i$ measurements taken from: SIMBAD, Bernat et al. (2010); Goldman et al. (2010); Lee et al. (2010); Reiners \& Basri (2010); Seifahrt et al. (2010); Smart et al. (2010); Del Burgo et al. (2009); Faherty et al. (2009); Leggett et al. (2009); Stephens et al. (2009); Looper et al. (2008); Reid et al. (2008); Reiners \& Basri (2008); Blake et al. (2007); Kendall et al. (2007); Looper et al. (2007); Reiners \& Basri (2007); Schmidt et al. (2007); Artigau et al. (2006); Henry et al. (2006); Morales-Calderón et al. (2006); Reid et al. (2006); Zapatero Osorio et al. (2006); Crifo et al. (2005); Cushing et al. (2005); Lodieu et al. (2005); Tinney et al. (2005); Golimowski et al. (2004); Vrba et al. (2004); Burgasser et al. (2003a); Cruz et al. (2003); Lépine et al. (2003, 2002).

list with the present survey raises the total number of studied ultracool dwarfs to 193 , with 13 detected to posses radio emission (see Fig. 4). Although this might suggest a detection rate of $\sim 6 \%$ in the ultracool dwarf regime, a more detailed analysis indicates otherwise. All but one of the detected ultracool dwarfs span the spectral type range M7-L3.5. Therefore, the detection rate over this spectral range is 12 confirmed sources from 134 observations, i.e., $\sim 9 \%$. In comparison, only one dwarf later than L3.5 was detected in 53 observations.

In Fig. 2, we plot the number distribution of cool dwarfs later than M0 (red line) observed in the radio domain compared to those with a detected radio signal (blue line) with respect to their spectral type. We include earlier type $\mathrm{M}$ dwarfs to show the overall trend in detection rate. The insert panel in the figure shows the detection rate as a function of spectral type, defined by $N_{\mathrm{r}} / N$, where $N_{\mathrm{r}}$ is the number of dwarfs with detected radio signal in a certain spectral type range; $N$ is the number of observed dwarfs in the same spectral type range. To estimate the uncertainty of the detection rate as a function of spectral type, we adopted the binomial distribution with the limits equivalent to $1 \sigma$ limits for a Gaussian distribution (see, e.g., the article of Burgasser et al. (2003b), where this model was applied to the statistics of T-dwarf binaries). There is a drop in detection rate from $\sim 50 \%$ at spectral type M4 to less than $15 \%$ for later spectral types. The drop may be due to the small sample of observed UCDs, especially those later than L0. Alternatively, the drop may have physical implications. We discuss these possible implications in Sect. 4.1.

The top panel in Fig. 3 shows the observed radio luminosity as a function of rotation velocity for the $\mathrm{M}$ and $\mathrm{L}$ dwarfs (most of them are observed by the VLA, mainly at 4.9 and $8.5 \mathrm{GHz}$ ). Solid circles are for the $\mathrm{M}$ and $\mathrm{L}$ dwarfs with detected strong 


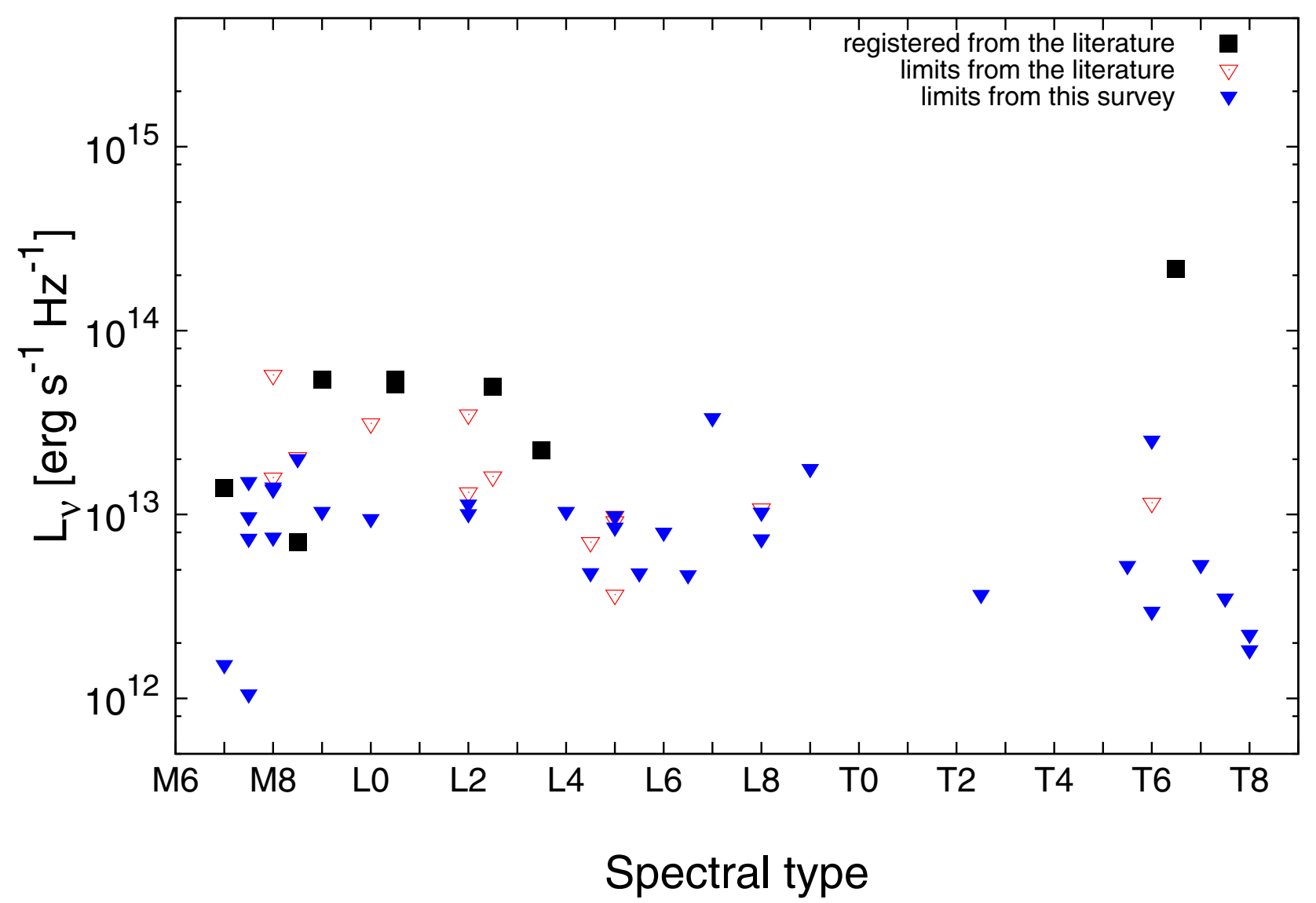

Fig. 1. Radio luminosity - spectral type plot for all ultracool dwarfs observed at $4.9 \mathrm{GHz}$. Filled triangles show the upper limits on radio luminosities of the dwarfs from the present survey, open triangles mark upper limits from the literature, and filled squares represent radio luminosities of detected dwarfs from the literature (Berger 2006; Burgasser \& Putman 2005; Antonova et al. 2007, 2008; Route \& Wolszczan 2012, and references therein).

Table 2. Upper limits on the radio flux and radio luminosity at $4.9 \mathrm{GHz}$ (present survey) and $8.5 \mathrm{GHz}$ (McLean et al. 2012) of the seven dwarfs that are present in both surveys.

\begin{tabular}{lccccc}
\hline \hline Name & Other name & $\begin{array}{c}F_{(4.9 \mathrm{GHz})} \\
\left(10^{-5} \mathrm{Jy}\right)\end{array}$ & $\begin{array}{c}L_{v, 4.9} \\
\left(\mathrm{erg} \mathrm{s}^{-1} \mathrm{~Hz}^{-1}\right)\end{array}$ & $\begin{array}{c}F_{(8.5 \mathrm{GHz})} \\
\left(10^{-5} \mathrm{Jy}\right)\end{array}$ & $\begin{array}{c}L_{\nu, 8.5} \\
\left(\mathrm{erg} \mathrm{s}^{-1} \mathrm{~Hz}^{-1}\right)\end{array}$ \\
\hline 2MASS J10481258-1120082 & GJ 3622 & $<6.3$ & $<1.53 \times 10^{12}$ & $<9.6$ & $<2.33 \times 10^{12}$ \\
2MASS J17571539+7042011 & LP 44-162 & $<8.1$ & $<1.51 \times 10^{13}$ & $<11.7$ & $<2.19 \times 10^{13}$ \\
2MASS J11554286-2224586 & LP 851-346 & $<6.6$ & $<7.43 \times 10^{12}$ & $<9.0$ & $<1.01 \times 10^{13}$ \\
2MASS J12505265-2121136 & & $<6.6$ & $<9.73 \times 10^{12}$ & $<7.2$ & $<1.06 \times 10^{13}$ \\
2MASS J09211410-2104446 & & $<7.2$ & $<1.14 \times 10^{13}$ & $<7.5$ & $<1.29 \times 10^{13}$ \\
2MASS J08283419-1309198 & & $<6.3$ & $<1.01 \times 10^{13}$ & $<6.6$ & $<1.06 \times 10^{13}$ \\
2MASSI J0700366+315726 & & $<4.2$ & $<8.01 \times 10^{12}$ & $<7.8$ & $<1.39 \times 10^{13}$ \\
\hline
\end{tabular}

Notes. We used the distances listed in Table1 to calculate $L_{v, 8.5}$.

radio signal, whilst open triangles represent the upper-limit of the luminosity of some $\mathrm{M}$ and $\mathrm{L}$ dwarfs. The relation between the luminosity and $v \sin i$ in this figure is not very clear. It seems that the number of $\mathrm{M}$ dwarfs possessing strong radio emission is more than the number of L dwarfs. Even for one individual dwarf, the detected radio luminosity can vary over a wide range (about 1-2 orders of magnitude) in a short observation period, which may suggest that the radio emission is strongly associated with its generation mechanism and may not be directly correlated with rotation rate.

The bottom panel in Fig. 3 illustrates the number distribution of all observed $\mathrm{M}$ (red line) and L (blue line) dwarfs as a function of rotation velocity $(v \sin i)$. The number distribution of
M dwarfs decreases with respect to $v \sin i$, perhaps caused by a stellar-wind-assisted magnetic braking. More observations are needed to exclude this selection effect. Also, since the majority of the data represent upper limits, no statistically significant conclusions can be inferred at present.

\section{Discussion}

In this section, we discuss the physical implication of our survey, including the influence of the radiation mechanism, the geometric effect, and the rotation of the dwarfs. A brief discussion on two other activity characteristics - X-ray and $\mathrm{H} \alpha$ emission from the radio observed sample - is also included in Appendix A. 


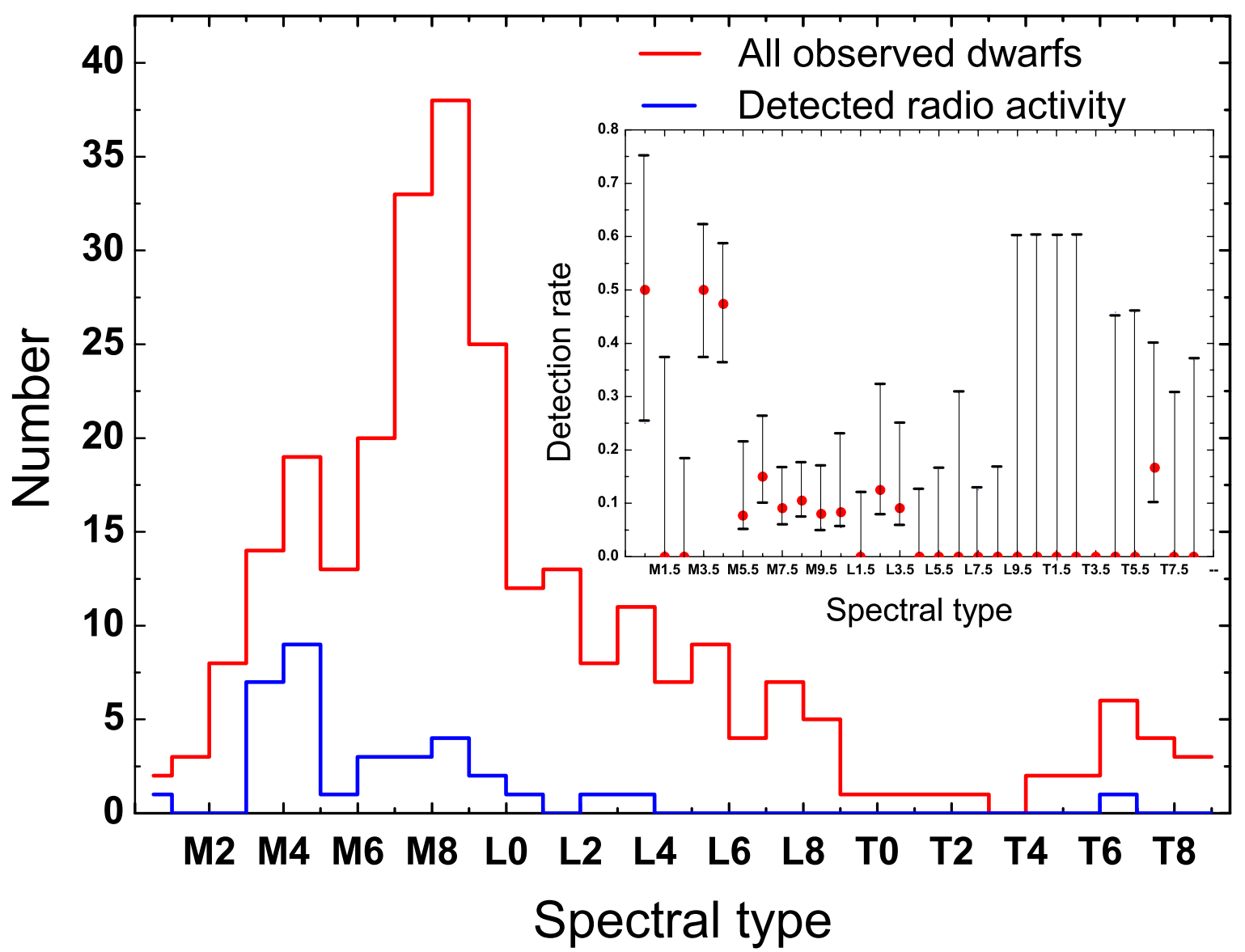

Fig. 2. Number distribution of observed dwarfs (red line) and dwarfs with detected radio signal (blue line) with respect to their spectral type. The insert panel shows the detection rate as a function of spectral type, defined by $N_{\mathrm{r}} / N$, where $N_{\mathrm{r}}$ is the number of dwarfs with detected radio signal in a certain spectral type range; $N$ is the number of observed dwarfs in the same spectral type range. In the spectral type range of M0-T8, the total number of observed dwarfs is 273 , while the number of dwarfs with detected radio signal is 34 . The zero detection rate around spectral type M2 is most likely due to the limited number of observations. Also shown are the uncertainties of the detection rates, estimated by adopting the binomial distribution at $1 \sigma$ level for the Gaussian distribution (see Sect. 3).

\subsection{Radiation mechanisms and geometric effect}

For 12 of the 13 radio active dwarfs a quiescent component of the radio emission was also detected. The T6.5 dwarf 2MASS J1047539+212423 was observed with the Arecibo single-dish, which has a low sensitivity to unpolarized quiescent emission, thus the presence of such a component is not confirmed, but it is likely. The nature of the quiescent emission is still debated. It could either be due to an incoherent process such, as gyrosynchrotron radiation, or depolarized electron cyclotron maser instability emission (Hallinan et al. 2008). On the other hand, the ECM is confirmed to be the dominant mechanism driving the bulk of the emission from five of the detected dwarfs (Hallinan et al. 2008; Berger et al. 2009; McLean et al. 2011). For the L3.5 dwarf 2MASS J00361617+1821104, Hallinan et al. (2008) showed that the radio emission is originally due to the ECM, but perhaps depolarized during propagation toward the observer. In addition, recent particle-in-cell numerical simulations by $\mathrm{Yu}$ et al. (2012) have shown that, under certain circumstances, maser emission can be intrinsically weakly polarized. The same mechanism can be applied to generate the bright, $100 \%$ polarized burst/flare detected from another ultracool dwarf - DENIS-P J104814.9-395604 (Burgasser \& Putman 2005).

Particle-in-cell simulations, which are based on the particlefield theory, indicate that a mildly relativistic or relativistic electron-beam-driven cyclotron maser can be an effective mechanism to release electromagnetic waves and heat the surrounding plasmas (Yu et al. 2012). Furthermore, from the diffusion process of the electrons in velocity space, a high-energy tail may be rapidly developed along the direction perpendicular to the magnetic field, which can eventually evolve to moderately or strongly relativistic electrons depending on the initial energy of the electron current, and contribute gyrosynchrotron radiation. This may lead to the appearance of a radio continuum and the deformation of the spectral energy distribution. Also, the simulations show that a series of discrete spectral lines can appear at certain frequency bands, which may increase the difficulty of finding the fundamental cyclotron frequency in the observed radio frequencies.

However, it is likely that the magnetic field inhomogeneity will smooth out these bands, thus producing a continuous spectrum. Therefore, it is possible that determining the cyclotron frequency and detecting radio emission from a larger number of UCDs may require observations over a wider frequency band.

In the case of beamed emission, the geometric selection may play an important role in determining the detection rate (see insert panel in Fig. 2). The probability $P$ to detect this emission can be expressed as $P=2 \theta /(4 \pi)$. The half solid angle of the radiation cone $\theta$ can be approximated by $\cos \theta \approx \frac{v_{\mathrm{e}}}{c}$, where $v_{\mathrm{e}}$ and $c$ are the velocity of electrons in a local region and the speed of light. 

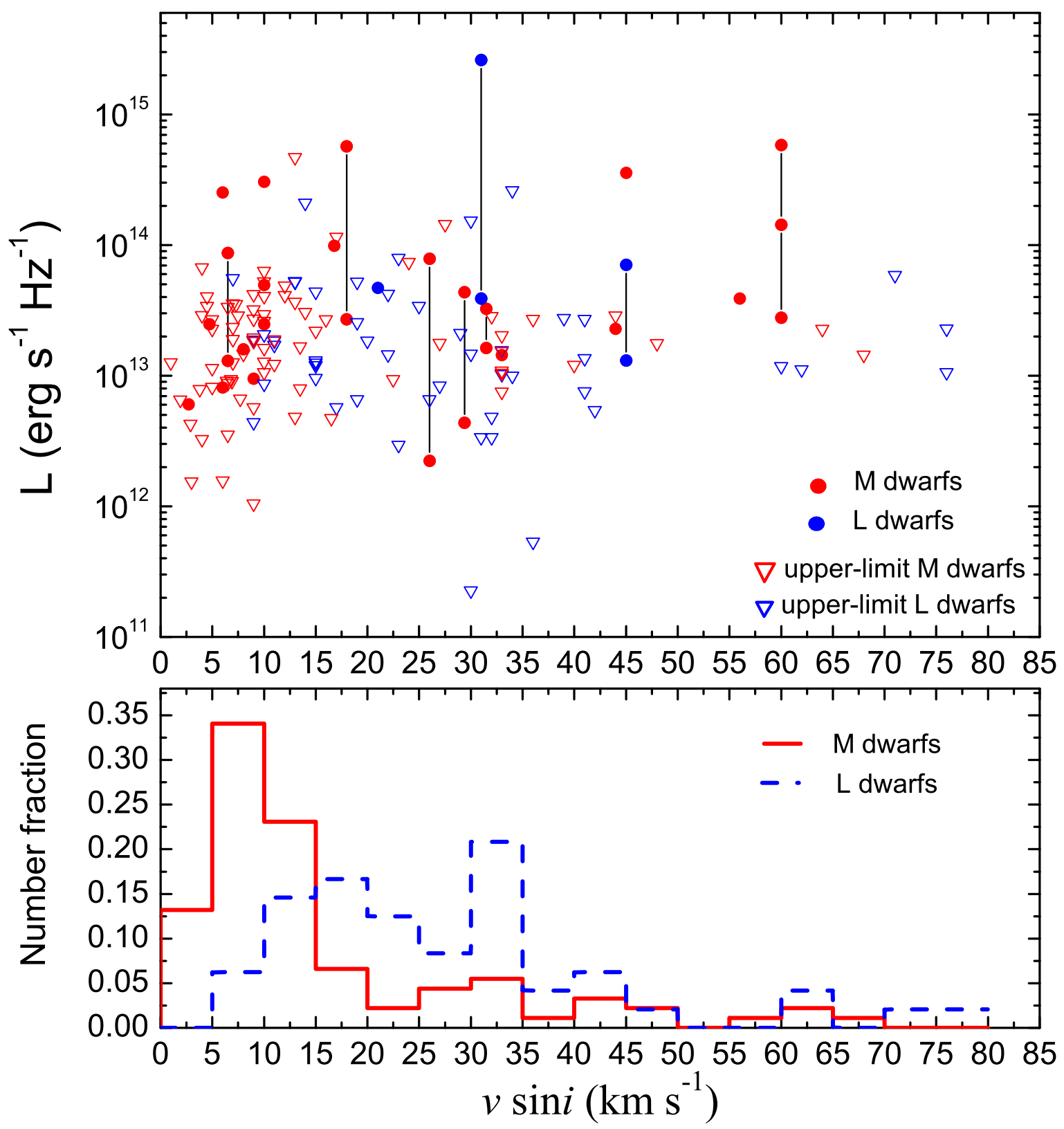

Fig. 3. Top panel: radio luminosity as a function of rotation velocity for the UCDs observed in the radio domain (mainly at 4.9 and $8.5 \mathrm{GHz}$ ). Red solid circles are for M dwarfs, the blue solid circles represent L dwarfs. The solid circles connected by black lines denote different measurements for the same target. Red open triangles denote the upper-limit of the luminosity of M dwarfs; blue open triangles stand for the upper-limit of the luminosity of L dwarfs. The data are mainly taken from Table 1, McLean et al. (2012), and references therein. Bottom panel: the number distribution of the observed dwarfs as a function of rotation velocity. The red solid line is for M dwarfs (normalized by the total number 91), the blue dashed line is for L dwarfs (normalized by the total number 48).

The current detection rate for dwarfs over spectral type M7-L3.5 is about $\sim 9 \%$, which may imply that we need a mildly relativistic electron beam with an average velocity of at least $0.8 c$.

The numerical simulations indicate that a relativistic electron beam can release more than $30 \%$ of its kinetic energy to electromagnetic energy in a relatively weak magnetic field (hundreds of Gauss) (Yu et al. 2012). So one tentative conclusion is that fast electron beams may be common even in cool objects, e.g., L dwarfs. The electrons may come from the magnetic field coupled ionization (or discharging) process in the atmosphere, as discussed in Helling et al. (2011). Alternatively, they can be associated with the internal activity of the ultracool dwarfs.

The ECM emission and/or geometric selection may be the explanation for the lack of detection of two particular dwarfs in our list - the M9 dwarf LHS 2924 and the M7 dwarf GJ 3622, both listed in SIMBAD as flare stars. LHS 2924 has detected $\mathrm{H} \alpha$ emission (see Table 1), magnetic field strength of $B f=$ $1.6 \pm 0.2 \mathrm{kG}$ (where $\mathrm{f}$ is the filling factor), and $v \sin i=10 \mathrm{~km} \mathrm{~s}^{-1}$ (Reiners \& Basri 2007). Depending on the inclination angle, the rotational velocity could be in the range 10 to $57 \mathrm{~km} \mathrm{~s}^{-1}$. Considering the presence of both chromospheric activity and kilogauss fields, it would not be surprising if radio emission was present as well. However, this is not the case. We have not detected this dwarf down to a $3 \sigma$ flux level of $6.3 \times 10^{-5} \mathrm{Jy}$ at $4.9 \mathrm{GHz}$. McLean et al. (2012) reported a $3 \sigma$ upper limit of $8.4 \times 10^{-5} \mathrm{Jy}$ at $8.5 \mathrm{GHz}$. The lack of detection at the higher frequency is not surprising considering the magnetic field strength measured. Detection at $4.9 \mathrm{GHz}$, on the other hand, should have 


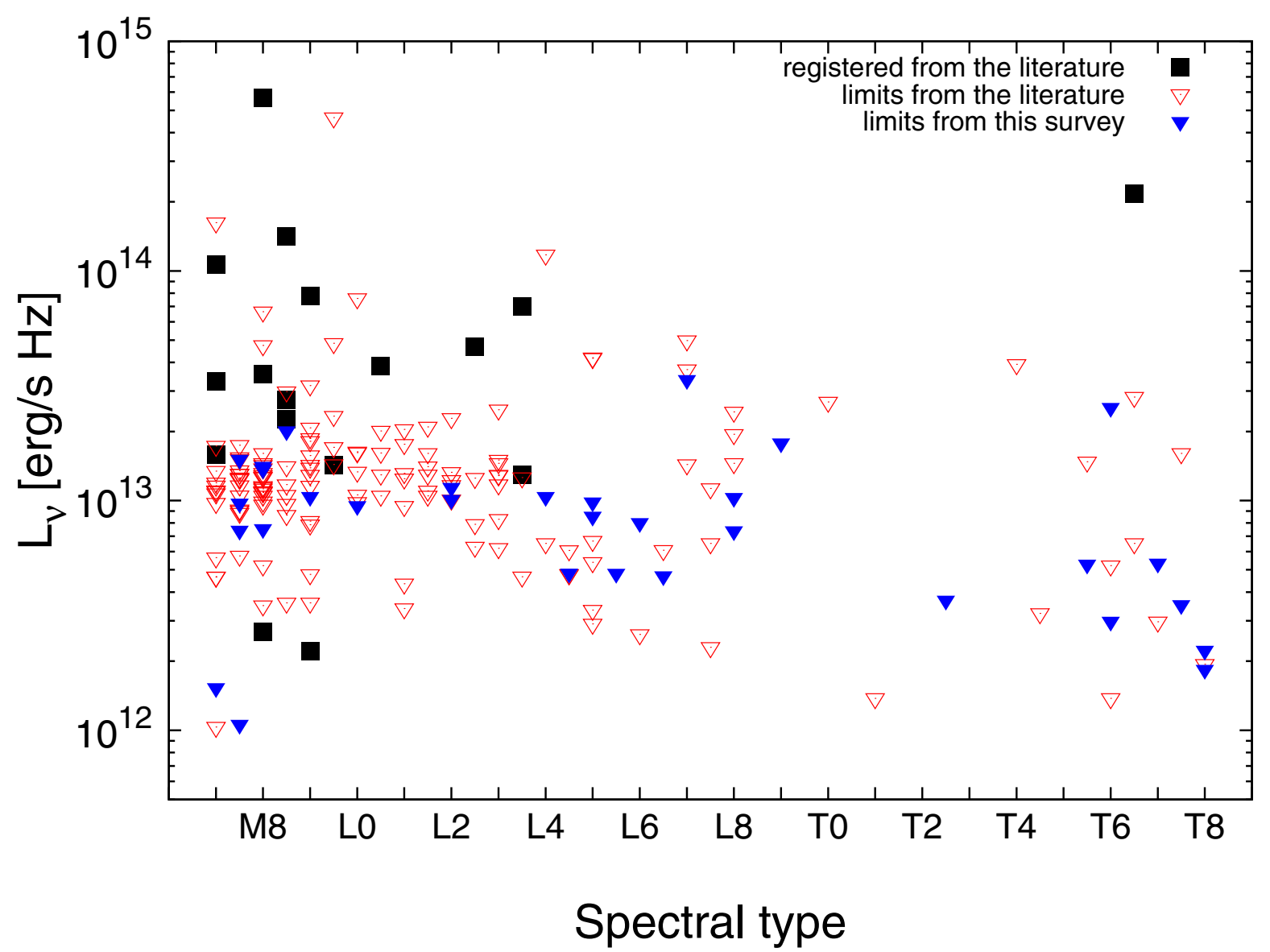

Fig. 4. Radio luminosity - spectral type plot for all ultracool dwarfs observed at radio frequencies. Filled triangles show the upper limits on radio luminosities of the dwarfs from the present survey. Open triangles mark upper limits from the literature and filled squares represent radio luminosities of detected dwarfs from the literature (McLean et al. 2012, and references therein).

been possible since the surface magnetic field is not less than $1600 \mathrm{G}$ (i.e., the cyclotron frequency exceeds $4.5 \mathrm{GHz}$ ). Thus, the absence of maser emission may be attributed to geometric effects since the beamed radio emission might never sweep in the direction of Earth during a full rotation of the UCD. However, for TVLM 513 the pulses have a duty cycle of less than $15 \%$, thus as an alternative explanation we may have missed the range of rotational phase during which pulsed emission is present.

The second dwarf, GJ 3622, has a detected $\mathrm{H} \alpha$ emission (see Table 1) and X-ray emission $\left(\log \left(L_{\mathrm{X}}\right)=25.96 \mathrm{erg} \mathrm{s}^{-1}\right)$, a magnetic field strength of $B f=600 \pm 200 \mathrm{G}$, and $v \sin i=3 \mathrm{~km} \mathrm{~s}^{-1}$ (Reiners \& Basri 2010; Lee et al. 2010; Schmitt \& Liefke 2004). The absence of radio emission from GJ 3622 can be explained by the fact that the surface magnetic field is only about $600 \mathrm{G}$, so that the maximal cyclotron frequency is $1.7 \mathrm{GHz}$, which is below our observation frequency.

The above considerations, however, cannot explain the absence of gyrosynchrotron emission, which has a wide directivity pattern and can be produced at frequencies well above the cyclotron frequency. Therefore, despite a strong magnetic field, the concentration and/or energy of accelerated electrons in the magnetospheres of the mentioned dwarfs may be insufficient to produce observable radio emission.

\subsection{Rotation}

All radio-detected dwarfs have been found to have high $v \sin i$ $\left(>20 \mathrm{~km} \mathrm{~s}^{-1}\right.$ ) values which may be interpreted as a dependence on rapid rotation (McLean et al. 2012). However, over 50 ultracool dwarfs with measured $v \sin i \gtrsim 15 \mathrm{~km} \mathrm{~s}^{-1}$ have not been detected as radio sources. It seems that fast rotation is a sufficient, but not necessary condition for late-M and L dwarfs to be radio-active. This tentative conclusion is consistent with the rotation and magnetic activity relation for a sample of M-dwarfs determined by measuring the equivalent widths of the $\mathrm{CaII} \mathrm{H}$ and $\mathrm{K}$ lines (Browning et al. 2010).

The radio emission from ultracool dwarfs can be strongly associated with their rotation rate in two ways. First, rotation plays a key role in the stellar dynamo, thus determining the magnetic field strength. Second, rotation can directly affect the process of particle acceleration. For the planets of the solar system, the energy of the accelerated particles can come from three sources: a) interaction of a magnetosphere with the solar wind (this process occurs at all planets and is especially important for the magnetospheres of the Earth, Uranus, and Neptune); b) interaction of a magnetosphere with a satellite (like in the Io-Jupiter system); c) centrifugal acceleration of the magnetospheric plasma.

The latter effect is caused by the fact that in a rigidly rotating magnetosphere, the centrifugal force exceeds gravity at some distance from the star/planet. This results in an accumulation of plasma and in an increase of the plasma pressure in those regions. Eventually, the plasma pressure exceeds the magnetic field pressure, so that the magnetic field lines become broken. As a result, the magnetic field at distances exceeding a certain radius does not rotate with the same rate as the star/planet; current sheets are then formed at the boundary between the inner 
(co-rotating) and outer parts of the magnetosphere. The electric fields in the mentioned current sheets, in turn, can accelerate electrons up to relativistic energies; thus the rotation energy is converted into particle energy (Andre et al. 1988; Linsky et al. 1992; Usov \& Melrose 1992; Leto et al. 2006).

The above process has been proven to be important for the magnetospheres of Jupiter and (to a lesser degree) Saturn. It is also proposed as the main particle acceleration mechanism for the radio-emitting star CU Virginis and other similar objects. Regarding the ultracool dwarfs, if a dwarf is not part of a binary system, the interaction with an external stellar wind is obviously absent. Satellite-induced radio emission can be recognized by its specific light curves reflecting both the dwarf's rotation and the satellite motion (Kuznetsov et al. 2012). The satellite-induced particle acceleration can be ruled out at least for some radio-emitting dwarfs, because this model cannot account for the strictly periodic emission pulses (which are typical of some dwarfs) nor for nearly constant quiescent emission (which is also observed). Thus, for an ultracool dwarf without close-in satellites, one probable particle acceleration mechanism is the centrifugal acceleration.

Recently, Nichols et al. (2012) have estimated the parameters of the electric currents produced in the rapidly rotating magnetospheres of ultracool dwarfs through angular velocity shear; it has been demonstrated that the power carried by these currents can be sufficient to provide the observed intensities of radio emission. An assumption in this model is that the spin and magnetic axes are co-aligned, which if the polarity were reversed, implies that only $\sim 50 \%$ of UCDs would have auroral and radio emission. Coupling this with the known radio-active duty cycle of $\sim 0.15$ phase for UCDs (Hallinan et al. 2008), similar to the active duty cycle of Jupiter of 0.14 phase (Higgins et al. 1996), could easily imply a very low detection rate.

Evidently, the efficiency of this mechanism is strongly dependent on the rotation rate: an increase of the rotation rate both increases the power transferred to the particles (Schrijver 2009) and shifts the acceleration region toward the stellar surface (for the rotation period of a few hours, the co-rotation radius is expected to equal just a few stellar radii). We propose the following explanation of non-detection of radio emission from the dwarfs LHS 2924 and GJ 3622: although the rotation rate of these dwarfs is relatively low, it is sufficient to produce the magnetic fields of kilogauss strength (indeed, the dynamo models predict that the dynamo effects saturates at relatively low rotation rates corresponding to $\mathrm{v}$ about $5 \mathrm{~km} \mathrm{~s}^{-1}$, so that a further increase in the rotation rate does not affect the magnetic field strength significantly (McLean et al. 2012)). At the same time, owing to the low rotation rate, the centrifugal acceleration mechanism at these dwarfs is too weak and cannot provide the necessary density and/or energy of accelerated electrons.

There is still no conclusive evidence on whether rotation or inclination angle is more important for detecting radio activity from UCDs. It might be the case that both play a significant role - the first for generating the emission and the latter for detecting it. The ultracool dwarfs with detected ECM emission are all found to be fast rotators with high inclination angles. The question whether detection of the radio emission from a larger sample depends on observing frequency may only be addressed via observations of individual targets over wide frequency bands with the upgraded JVLA and ATCA.

There are currently several ongoing modeling projects that aim at a better understanding of the emission process that produces the periodic radio bursts with high brightness temperature and polarization degree in UCDs. Recent computations indicate that the rotation-modulated ECM emission can interpret the radio light curve from the M8.5 dwarf TVLM 513-46546 (Yu et al. 2011), therefore the properties of the radio emission region can be determined. Future work will focus on the combination between different magnetic field topology and a particle simulation to reproduce the local radio emission region.

Kuznetsov et al. (2012) presented results for the same dwarf where the emission properties were similar to the auroral radio emission of the magnetized planets of the solar system via the electron-cyclotron maser instability. Two models were considered where the emission was caused by interaction with a satellite or derived from a narrow sector of active longitudes. It was found that the model of emission from an active sector is able to qualitatively reproduce the main features of the radio light curves; the magnetic dipole needs to be highly tilted (by about $60^{\circ}$ ) with respect to the rotation axis. For the most often observationally studied object, TVLM 513-46546, the model of the satellite-induced emission was inconsistent with the observations.

\subsection{Other possibilities}

Another possible explanation for the lack of detected radio emission could be long-term variability. To date, there are several ultracool dwarfs whose radio emission varies considerably over long periods, with emission levels dropping below the detection limit in some cases (Antonova et al. 2007; Berger et al. 2010; McLean et al. 2012).

Long-term variability, however, requires systematic monitoring of large sample of targets. Furthermore, full rotational phase coverage should be obtained to account for the narrow beaming of electron-cyclotron maser emission.

Acknowledgements. We gratefully acknowledge funding for this project by the Bulgarian National Science Fund (contract No DDVU02/40/2010). Research at Armagh Observatory is grant-aided by the N. Ireland Department of Culture, Arts and Leisure. This research has made use of the SIMBAD database, operated at CDS, Strasbourg, France. AKU, SYU \& JGD thank the Leverhulme Trust for their support of this project.

\section{References}

Andre, P., Montmerle, T., Feigelson, E. D., Stine, P. C., \& Klein, K.-L. 1988, ApJ, 335, 940

Antonova, A., Doyle, J. G., Hallinan, G., Golden, A., \& Koen, C. 2007, A\&A, 472,257

Antonova, A., Doyle, J. G., Hallinan, G., Bourke, S., \& Golden, A. 2008, A\&A, 487,317

Artigau, É., Doyon, R., Lafrenière, D., et al. 2006, ApJ, 651, L57

Audard, M., Osten, R. A., Brown, A., et al. 2007, A\&A, 471, L63

Berger, E. 2006, ApJ, 648, 629

Berger, E., Rutledge, R. E., Phan-Bao, N., et al. 2009, ApJ, 695, 310

Berger, E., Basri, G., Fleming, T. A., et al. 2010, ApJ, 709, 332

Bernat, D., Bouchez, A. H., Ireland, M., et al. 2010, ApJ, 715, 724

Blake, C. H., Charbonneau, D., White, R. J., Marley, M. S., \& Saumon, D. 2007, ApJ, 666, 1198

Browning, M. K., Basri, G., Marcy, G. W., West, A. A., \& Zhang, J. 2010, AJ, 139,504

Burgasser, A. J., \& Putman, M. E. 2005, ApJ, 626, 486

Burgasser, A. J., Kirkpatrick, J. D., Liebert, J., \& Burrows, A. 2003a, ApJ, 594, 510

Burgasser, A. J., Kirkpatrick, J. D., Reid, I. N., et al. 2003b, ApJ, 586, 512

Crifo, F., Phan-Bao, N., Delfosse, X., et al. 2005, A\&A, 441, 653

Cruz, K. L., Reid, I. N., Liebert, J., Kirkpatrick, J. D., \& Lowrance, P. J. 2003 , AJ, 126, 2421

Cruz, K. L., Reid, I. N., Kirkpatrick, J. D., et al. 2007, AJ, 133, 439

Cushing, M. C., Rayner, J. T., \& Vacca, W. D. 2005, ApJ, 623, 1115

Del Burgo, C., Martín, E. L., Zapatero Osorio, M. R., \& Hauschildt, P. H. 2009, A\&A, 501, 1059 
Doyle, J. G., Antonova, A., Marsh, M. S., et al. 2010, A\&A, 524, A15 Faherty, J. K., Burgasser, A. J., Cruz, K. L., et al. 2009, AJ, 137, 1 Fleming, T. A., Giampapa, M. S., Schmitt, J. H. M. M., \& Bookbinder, J. A. 1993, ApJ, 410, 387

Goldman, B., Marsat, S., Henning, T., Clemens, C., \& Greiner, J. 2010, MNRAS, 405,1140

Golimowski, D. A., Leggett, S. K., Marley, M. S., et al. 2004, AJ, 127, 3516

Grosso, N., Briggs, K. R., Güdel, M., et al. 2007, A\&A, 468, 391

Guedel, M. \& Benz, A. O. 1993, ApJ, 405, L63

Hallinan, G., Antonova, A., Doyle, J. G., et al. 2006, ApJ, 653, 690

Hallinan, G., Bourke, S., Lane, C., et al. 2007, ApJ, 663, L25

Hallinan, G., Antonova, A., Doyle, J. G., et al. 2008, ApJ, 684, 644

Helling, C., Jardine, M., Witte, S., \& Diver, D. A. 2011, ApJ, 727, 4

Henry, T. J., Jao, W.-C., Subasavage, J. P., et al. 2006, AJ, 132, 2360

Higgins, C. A., Carr, T. D., \& Reyes, F. 1996, Geophys. Res. Lett., 23, 2653

Kendall, T. R., Jones, H. R. A., Pinfield, D. J., et al. 2007, MNRAS, 374, 445

Kuznetsov, A. A., Doyle, J. G., Yu, S., et al. 2012, ApJ, 746, 99

Lee, K.-G., Berger, E., \& Knapp, G. R. 2010, ApJ, 708, 1482

Leggett, S. K., Cushing, M. C., Saumon, D., et al. 2009, ApJ, 695, 1517

Lépine, S., Rich, R. M., Neill, J. D., Caulet, A., \& Shara, M. M. 2002, ApJ, 581, L47

Lépine, S., Rich, R. M., \& Shara, M. M. 2003, AJ, 125, 1598

Leto, P., Trigilio, C., Buemi, C. S., Umana, G., \& Leone, F. 2006, A\&A, 458, 831

Linsky, J. L., Drake, S. A., \& Bastian, T. S. 1992, ApJ, 393, 341

Lodieu, N., Scholz, R.-D., McCaughrean, M. J., et al. 2005, A\&A, 440, 1061

Looper, D. L., Kirkpatrick, J. D., \& Burgasser, A. J. 2007, AJ, 134, 1162

Looper, D. L., Kirkpatrick, J. D., Cutri, R. M., et al. 2008, ApJ, 686, 528

McLean, M., Berger, E., Irwin, J., Forbrich, J., \& Reiners, A. 2011, ApJ, 741, 27

McLean, M., Berger, E., \& Reiners, A. 2012, ApJ, 746, 23

Morales-Calderón, M., Stauffer, J. R., Kirkpatrick, J. D., et al. 2006, ApJ, 653, 1454

Nichols, J. D., Burleigh, M. R., Casewell, S. L., et al. 2012, ApJ, 760, 59

Reid, I. N., Lewitus, E., Allen, P. R., Cruz, K. L., \& Burgasser, A. J. 2006, AJ, 132, 891

Reid, I. N., Cruz, K. L., Kirkpatrick, J. D., et al. 2008, AJ, 136, 1290

Reiners, A., \& Basri, G. 2007, ApJ, 656, 1121

Reiners, A., \& Basri, G. 2008, ApJ, 684, 1390

Reiners, A., \& Basri, G. 2010, ApJ, 710, 924

Route, M., \& Wolszczan, A. 2012, ApJ, 747, L22

Schmitt, J. H. M. M., \& Liefke, C. 2004, A\&A, 417, 651

Schmidt, S. J., Cruz, K. L., Bongiorno, B. J., Liebert, J., \& Reid, I. N. 2007, AJ, 133,2258

Schrijver, C. J. 2009, ApJ, 699, L148

Seifahrt, A., Reiners, A., Almaghrbi, K. A. M., \& Basri, G. 2010, A\&A, 512, A37

Smart, R. L., Ioannidis, G., Jones, H. R. A., Bucciarelli, B., \& Lattanzi, M. G. 2010, A\&A, 514, A84

Stelzer, B., Alcalá, J., Biazzo, K., et al. 2012, A\&A, 537, A94

Stephens, D. C., Leggett, S. K., Cushing, M. C., et al. 2009, ApJ, 702, 154

Tinney, C. G., Burgasser, A. J., Kirkpatrick, J. D., \& McElwain, M. W. 2005, AJ, 130, 2326

Treumann, R. A. 2006, A\&ARv, 13, 229

Usov, V. V., \& Melrose, D. B. 1992, ApJ, 395, 575
Vrba, F. J., Henden, A. A., Luginbuhl, C. B., et al. 2004, AJ, 127, 2948

Yu, S., Hallinan, G., Doyle, J. G., et al. 2011, A\&A, 525, A39

Yu, S., Doyle, J. G., Kuznetsov, A., et al. 2012, ApJ, 752, 60

Zapatero Osorio, M. R., Martín, E. L., Bouy, H., et al. 2006, ApJ, 647, 1405

\section{Appendix A: Activity in $\mathrm{H} \alpha$ and $\mathrm{X}$-rays of the sample of radio-observed dwarfs}

In Table A.1 we list all ultracool dwarfs observed in the radio domain, their distances, $v \sin i$, radio fluxes, and luminosities/upper limits in $\mathrm{H} \alpha$, X-rays, and the radio. About $60 \%$ of the radio-observed dwarfs also have observations in $\mathrm{H} \alpha$, while only $\approx 18 \%$ were observed in X-rays. Figures A.1 and A.2 show the $\mathrm{H} \alpha$ and X-ray quiescent luminosities as a function of spectral type. Despite the trend of lower $L_{\mathrm{H} \alpha} / L_{\mathrm{bol}}$ with later spectral type, only three UCDs of type $\leq \mathrm{L} 7.5$ have upper limits on their $\mathrm{H} \alpha$ luminosities. Toward the later spectral types, only one dwarf has a marginal $\mathrm{H} \alpha$ detection - the one detected with Arecibo, the T6.5 dwarf 2MASS J1047+21 (Route \& Wolszczan 2012; Burgasser et al. 2003a). However, its activity level is comparable to that of early-L dwarfs. Overall, these results point to chromospheric activity present throughout the $\mathrm{L}$ spectral type and possibly as far as late-T types.

For late-type, main-sequence active stars (and especially active $M$ dwarfs), there is an empirical correlation between the observed $X$-ray and radio emission $\left(L_{\mathrm{R}} / L_{\mathrm{X}} \approx 10^{-15.5}\right)$ found by Guedel \& Benz (1993). The suggestion is that the same population of relativistic electrons is responsible for both emissions the spiraling motions of the electrons in the magnetic field give rise to incoherent radio emission prior to heating the coronal plasma, which leads to thermal X-ray emission. However, for the ultracool dwarfs this correlation is found to be violated by several orders of magnitude (Berger et al. 2010, and references there in). For UCDs with observations in both the X-ray and radio domains, Stelzer et al. (2012) suggested that they may be separated into two groups: X-ray flaring but radio-faint objects that are also slow rotators, and $\mathrm{X}$-ray-faint but strong radio-bursting dwarfs with fast rotation. However, there are several dwarfs with $v \sin i$ in the range $15-60 \mathrm{~km} \mathrm{~s}^{-1}$ that do not follow this pattern. For example, the L2 dwarf Kelu-1 (with $v \sin i=60 \mathrm{~km} \mathrm{~s}^{-1}$ ) has been reported to only possess X-ray emission (Audard et al. 2007). As seen from Table A.1 and Fig. A.2, the sample of dwarfs observed both in radio and $\mathrm{X}$-rays is too small to allow any significant conclusions. 


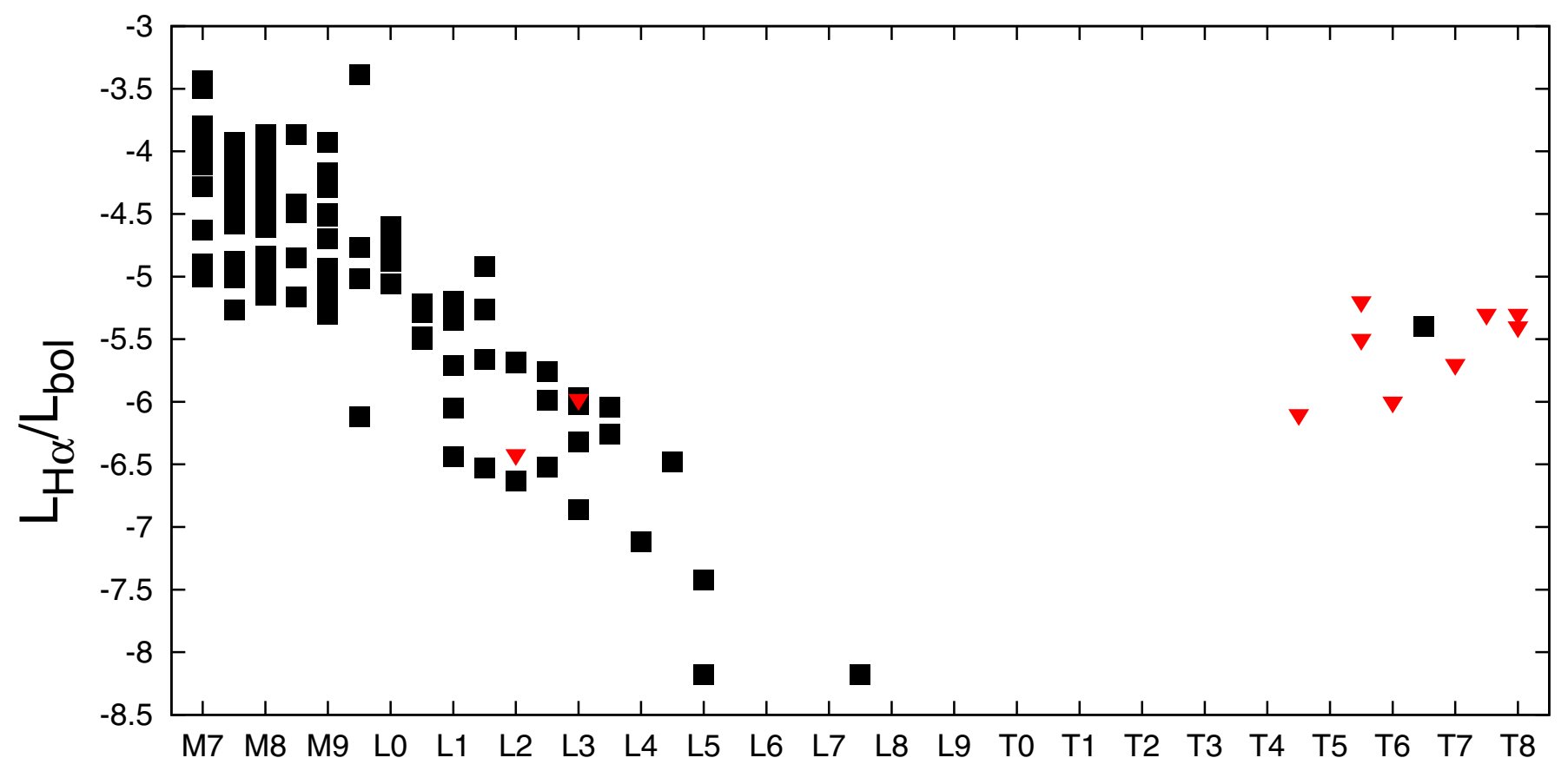

\section{Spectral type}

Fig. A.1. H $\alpha$ luminosity - spectral type for all ultracool dwarfs observed at radio frequencies. Squares represent luminosities while triangles show upper limits. Data are taken from the literature (McLean et al. 2012; Burgasser et al. 2003a; Berger et al. 2010, and references therein).

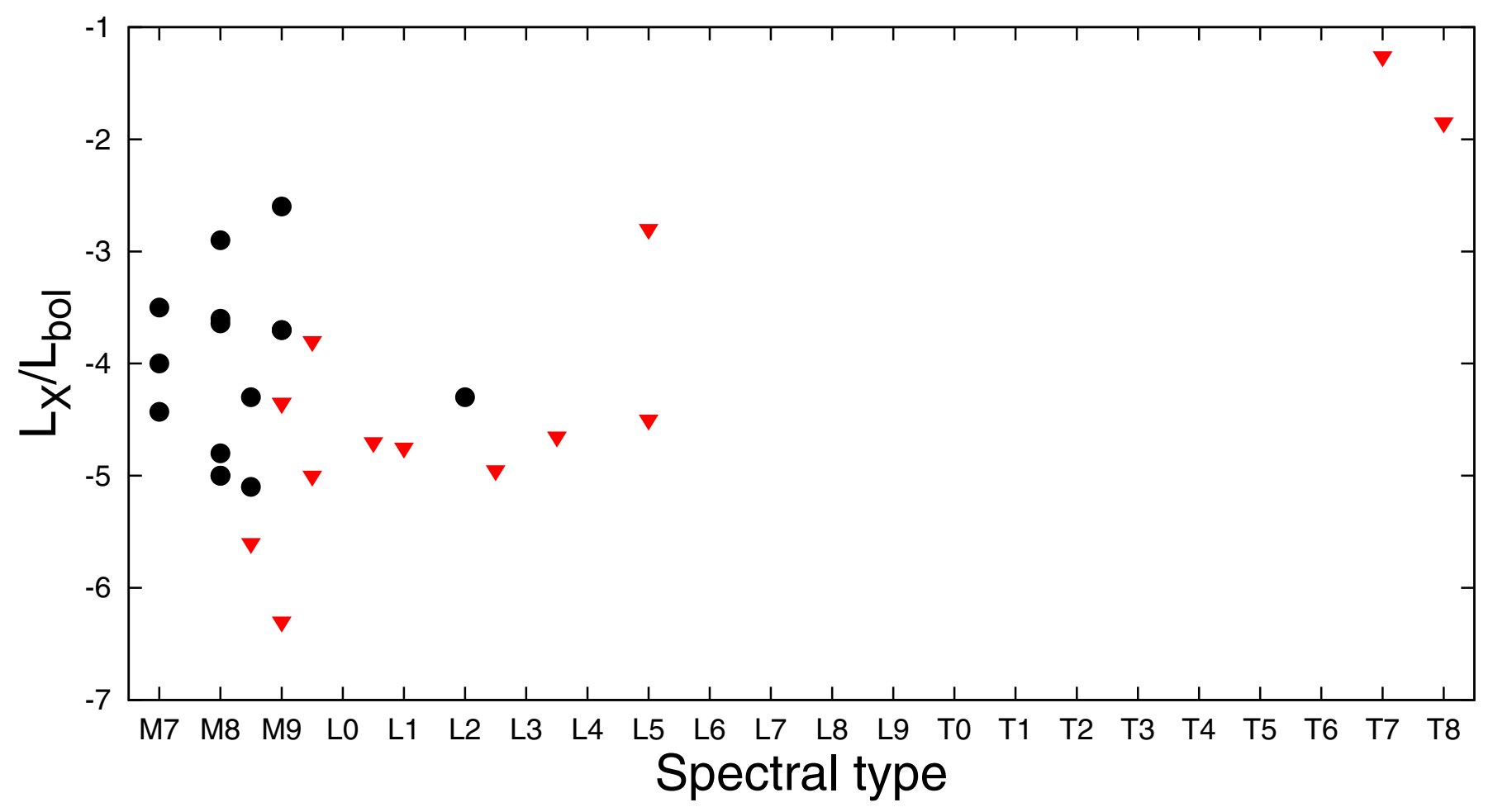

Fig. A.2. X-ray luminosity - spectral type for all ultracool dwarfs observed at radio frequencies. Circles represent luminosities while triangles show upper limits. Data are taken from the literature (McLean et al. 2012; Stelzer et al. 2012; Berger et al. 2010; Grosso et al. 2007; Schmitt \& Liefke 2004; Burgasser et al. 2003a; Fleming et al. 1993, and references therein). 
A. Antonova et al.: Volume-limited radio survey of ultracool dwarfs

Table A.1. Activity characteristics of all ultracool dwarfs observed at radio frequencies.

\begin{tabular}{|c|c|c|c|c|c|c|c|c|c|}
\hline $\begin{array}{l}\text { Name } \\
(2 \text { MASS J) }\end{array}$ & Other name & Sp.T. & $\begin{array}{c}d \\
(\mathrm{pc})\end{array}$ & $\begin{array}{c}v \sin i \\
\left(\mathrm{~km} \mathrm{~s}^{-1}\right)\end{array}$ & $\begin{array}{l}L_{\text {bol }} \\
\left(L_{\odot}\right)\end{array}$ & $L_{\mathrm{H} \alpha} / L_{\mathrm{bol}}$ & $L_{\mathrm{X}} / L_{\mathrm{bol}}$ & $\begin{array}{c}F_{v} \\
(\mu \mathrm{Jy})\end{array}$ & $\begin{array}{c}L_{V} \\
\left(\mathrm{erg} \mathrm{s}^{-1} \mathrm{~Hz}^{-1}\right)\end{array}$ \\
\hline $10481258-1120082$ & GJ 3622 & M7 & 4.5 & 3.0 & -3.16 & -4.63 & -4.43 & $\begin{array}{l}<63 \\
<96\end{array}$ & $\begin{array}{l}<1.53 \times 10^{12} \\
<2.33 \times 10^{12}\end{array}$ \\
\hline $0435161-160657$ & LP 775-31 & M7.0 & 9 & $\ldots$ & -3.59 & -4.28 & $\ldots$ & $<48$ & $<4.65 \times 10^{12}$ \\
\hline 0440232-053008 & LP 655-48 & M7.0 & 10 & 16.5 & -3.62 & -3.80 & $\ldots$ & $<39$ & $<4.67 \times 10^{12}$ \\
\hline $0752239+161215$ & LP $423-31$ & M7.0 & 11 & 9 & -3.56 & -3.44 & $\ldots$ & $<39$ & $<5.65 \times 10^{12}$ \\
\hline $1456383-280947$ & GJ 3877 & M7.0 & 7 & 8 & -3.29 & -4.02 & -4.0 & $270 \pm 40$ & $1.58 \times 10^{13}$ \\
\hline $1634216+571008$ & GJ $630.1 \mathrm{~B}$ & M7.0 & 16 & $\ldots$ & -3.13 & $\ldots$ & $\ldots$ & $<530$ & $<1.62 \times 10^{14}$ \\
\hline $1655352-082340$ & VB 8 & M7.0 & 6 & 9 & -3.21 & $\begin{array}{c}-5.0 \\
-2.85(\mathrm{f})\end{array}$ & $\begin{array}{c}-3.5 \\
-2.85(f)\end{array}$ & $<24$ & $<1.03 \times 10^{12}$ \\
\hline $0741068+173845$ & LHS 1937 & M7.0 & 18 & 10 & -3.17 & -4.10 & $\ldots$ & $<75$ & $<2.91 \times 10^{13}$ \\
\hline $0818580+233352$ & & M7.0 & 19 & 4.5 & -3.19 & -4.11 & $\ldots$ & $<78$ & $<3.37 \times 10^{13}$ \\
\hline 0952219-192431 & & M7.0 & 30 & 6 & -3.08 & -3.94 & $\ldots$ & $\begin{array}{c}<69 \\
233 \pm 15\end{array}$ & $\begin{array}{c}<7.43 \times 10^{13} \\
2.51 \times 10^{14}\end{array}$ \\
\hline 1141440-223215 & & M7.0 & 22 & 10 & -3.25 & -4.90 & $\ldots$ & $<108$ & $<6.25 \times 10^{13}$ \\
\hline $1314203+132001 \mathrm{~A}$ & & M7.0 & 16 & 45 & -3.17 & -3.97 & $\ldots$ & $1156 \pm 15$ & $3.54 \times 10^{14}$ \\
\hline $1354087+084608$ & & M7.0 & 17 & $\ldots$ & -3.79 & $\ldots$ & $\ldots$ & $<105$ & $<3.63 \times 10^{13}$ \\
\hline $13564148+4342587$ & LP 220-13 & M7.0 & 16 & 14 & -3.59 & -3.92 & $\ldots$ & $<99$ & $<3.03 \times 10^{13}$ \\
\hline $1534570-141848$ & 2MUCDa11346 & M7.0 & 11 & 10 & -3.34 & -4.01 & $\ldots$ & $<87$ & $<1.26 \times 10^{13}$ \\
\hline $2337383-125027$ & LP 763-3 & M7.0 & 19 & $\ldots$ & -2.89 & -3.50 & $\ldots$ & $<84$ & $<3.63 \times 10^{13}$ \\
\hline $17571539+7042011$ & LP 44-162 & M7.5 & 12.5 & 33 & -3.48 & -5.01 & $\ldots$ & $\begin{array}{l}<81 \\
<117\end{array}$ & $\begin{array}{l}<1.51 \times 10^{13} \\
<2.19 \times 10^{13}\end{array}$ \\
\hline $11554286-2224586$ & LP 851-346 & M7.5 & 9.7 & 33 & -3.30 & $\begin{array}{l}-4.58 \\
-4.58\end{array}$ & $\ldots$ & $\begin{array}{l}<66 \\
<90\end{array}$ & $\begin{array}{l}<7.43 \times 10^{12} \\
<1.01 \times 10^{13}\end{array}$ \\
\hline 05395200-0059019 & SDSS J0539-0059 & M7.5 & 3.84 & $\ldots$ & . & $\ldots$ & $\ldots$ & $<60$ & $<1.06 \times 10^{12}$ \\
\hline $12505265-2121136$ & DENIS J1250-2121 & M7.5 & 11 & $\ldots$ & -3.25 & $\ldots$ & $\ldots$ & $\begin{array}{l}<66 \\
<72\end{array}$ & $\begin{array}{l}<9.73 \times 10^{12} \\
<1.04 \times 10^{13}\end{array}$ \\
\hline 0148386-302439 & & M7.5 & 18 & 48 & -3.67 & -4.35 & $\ldots$ & $<45$ & $<1.74 \times 10^{13}$ \\
\hline $0331302-304238$ & LP $888-18$ & M7.5 & 12 & $<3$ & -3.70 & -4.07 & $\ldots$ & $<72$ & $<1.51 \times 10^{13}$ \\
\hline 0417374-080000 & & M7.5 & 17 & 7 & -3.72 & -4.32 & $\ldots$ & $<36$ & $<1.25 \times 10^{13}$ \\
\hline 0429184-312356 & & M7.5 & 10 & $<3$ & -3.70 & -3.93 & $\ldots$ & $<48$ & $<5.74 \times 10^{12}$ \\
\hline $1521010+505323$ & NLTT 40026 & M7.5 & 16 & 40 & -3.70 & -4.88 & $\ldots$ & $<39$ & $<1.19 \times 10^{13}$ \\
\hline $0351000-005244$ & GJ 3252 & M7.5 & 15 & 6.5 & -3.06 & -4.16 & $\ldots$ & $<123$ & $<3.31 \times 10^{13}$ \\
\hline $1006319-165326$ & LP 789-23 & M7.5 & 16 & 16 & -3.28 & -4.22 & $\ldots$ & $<87$ & $<2.67 \times 10^{13}$ \\
\hline $1246517+314811$ & LHS 2632 & M7.5 & 18 & 7.3 & -3.25 & -5.27 & $\ldots$ & $<90$ & $<3.49 \times 10^{13}$ \\
\hline $1253124+403403$ & LP $218-8$ & M7.5 & 17 & 9 & -3.29 & -4.27 & $\ldots$ & $<78$ & $<2.70 \times 10^{13}$ \\
\hline $1332244-044112$ & & M7.5 & 21 & 9 & -3.18 & -4.37 & $\ldots$ & $<60$ & $<3.17 \times 10^{13}$ \\
\hline $1507277-200043$ & & M7.5 & 14 & 64 & -3.61 & -4.47 & $\ldots$ & $<96$ & $<2.25 \times 10^{13}$ \\
\hline $1546054+374946$ & & M7.5 & 20 & 10 & -3.25 & -3.98 & $\ldots$ & $<84$ & $<4.02 \times 10^{13}$ \\
\hline $2331217-274950$ & & M7.5 & 15 & 9 & -3.06 & -4.03 & $\cdots$ & $<72$ & $<1.94 \times 10^{13}$ \\
\hline $04351455-1414468$ & & M8 & 14 & $\ldots$ & $\ldots$ & $\ldots$ & $\cdots$ & $<60$ & $<1.41 \times 10^{13}$ \\
\hline $02150802-3040011$ & LP885-35 & M8 & 12.37 & $\begin{array}{l}\cdots \\
\cdots\end{array}$ & -3.55 & $\begin{array}{l}\cdots \\
\cdots\end{array}$ & $\begin{array}{l}\cdots \\
\cdots\end{array}$ & $<75$ & $<1.37 \times 10^{13}$ \\
\hline $05392474+4038437$ & LSR J0539+4038 & M8 & 10 & $\cdots$ & $\ldots$ & $\cdots$ & $\begin{array}{l}\cdots \\
\cdots\end{array}$ & $<63$ & $<7.54 \times 10^{12}$ \\
\hline $0019262+461407$ & & M8.0 & 19 & 68 & -3.80 & -4.51 & $\cdots$ & $<33$ & $<1.43 \times 10^{13}$ \\
\hline $0350573+181806$ & LP $413-53$ & M8.0 & 23 & 4 & -3.82 & $\ldots$ & $\begin{array}{l}\cdots \\
\cdots\end{array}$ & $<105$ & $<6.65 \times 10^{13}$ \\
\hline $0436103+225956$ & & M8.0 & 140 & ... & -2.62 & $\begin{array}{l}\cdots \\
\cdots\end{array}$ & $\begin{array}{l}\cdots \\
\cdots\end{array}$ & $<45$ & $<1.06 \times 10^{15}$ \\
\hline $0517376-334902$ & & M8.0 & 15 & $\ddot{8}$ & -3.82 & -4.42 & $\begin{array}{l}\cdots \\
\cdots\end{array}$ & $<54$ & $<1.45 \times 10^{13}$ \\
\hline $1016347+275149$ & LHS 2243 & M8.0 & 16 & $<3$ & -3.65 & -3.87 & $\begin{array}{l}\cdots \\
\ldots\end{array}$ & $<45$ & $<1.38 \times 10^{13}$ \\
\hline $1048146-395606$ & DENIS 1048 & M8.0 & 4 & 18 & -3.39 & -5.15 & -5.0 & $\begin{array}{c}140 \pm 40 \\
29600 \pm 100\end{array}$ & $\begin{array}{l}2.68 \times 10^{12} \\
5.67 \times 10^{14}\end{array}$ \\
\hline $1139511-315921$ & & M8.0 & 20 & $\ldots$ & -3.39 & $\ldots$ & -4.8 & $<99$ & $<4.74 \times 10^{13}$ \\
\hline $1534570-141848$ & & M8.0 & 11 & 10 & -3.39 & -4.01 & $\ldots$ & $<111$ & $<1.61 \times 10^{13}$ \\
\hline $1843221+404021$ & GJ 4073 & M8.0 & 14 & 5 & $\begin{array}{l}-3.51 \\
-3.09\end{array}$ & $\begin{array}{l}-4.11 \\
-4.11\end{array}$ & -3.64 & $\begin{array}{l}<48 \\
<96\end{array}$ & $\begin{array}{l}<1.13 \times 10^{13} \\
<2.25 \times 10^{13}\end{array}$ \\
\hline $1916576+050902$ & VB 10 & M8.0 & 6 & 6.5 & -3.35 & $\begin{array}{c}-4.9 \\
-4.4(f)\end{array}$ & $\begin{array}{l}-5.0 \\
-4.1(f)\end{array}$ & $<81$ & $<3.49 \times 10^{12}$ \\
\hline $2037071-113756$ & & M8.0 & 17 & $<3$ & -3.74 & -5.02 & $\ldots$ & $<33$ & $<1.14 \times 10^{13}$ \\
\hline $0027559+221932$ & LP 349-25 B & M8.0 & 10 & 56 & -3.12 & -4.53 & $\ldots$ & $323 \pm 14$ & $3.86 \times 10^{13}$ \\
\hline $0248410-165121$ & & M8.0 & 17 & $<3$ & -3.45 & -4.25 & $\ldots$ & $<81$ & $<2.80 \times 10^{13}$ \\
\hline $0320596+185423$ & LP $412-31$ & M8.0 & 15 & 15 & -3.26 & -3.87 & $\begin{array}{c}-3.6 \\
-0.65(f)\end{array}$ & $<81$ & $<2.18 \times 10^{13}$ \\
\hline
\end{tabular}

References. Data taken from this paper; McLean et al. (2012); Berger et al. (2010); Route \& Wolszczan (2012); Stelzer et al. (2012); Grosso et al. (2007); Schmitt \& Liefke (2004); Burgasser et al. (2003a); Fleming et al. (1993) and references therein. 
Table A.1. continued.

\begin{tabular}{|c|c|c|c|c|c|c|c|c|c|}
\hline $\begin{array}{l}\text { Name } \\
(2 M A S S ~ J)\end{array}$ & Other name & Sp.T. & $\begin{array}{c}d \\
(\mathrm{pc})\end{array}$ & $\begin{array}{c}v \sin i \\
\left(\mathrm{~km} \mathrm{~s}^{-1}\right)\end{array}$ & $\begin{array}{l}L_{\text {bol }} \\
\left(L_{\odot}\right)\end{array}$ & $L_{\mathrm{H} \alpha} / L_{\mathrm{bol}}$ & $L_{\mathrm{X}} / L_{\mathrm{bol}}$ & $\begin{array}{c}F_{v} \\
(\mu \mathrm{Jy})\end{array}$ & $\begin{array}{c}L_{V} \\
\left(\mathrm{erg} \mathrm{s}^{-1} \mathrm{~Hz}^{-1}\right) \\
\end{array}$ \\
\hline $0544115-243301$ & & M8.0 & 19 & $<3$ & -3.33 & -4.12 & .. & $<63$ & $<2.72 \times 10^{13}$ \\
\hline 0629235-024851B & GJ 234 B & M8.0 & 4 & $\ldots$ & -3.00 & $\ldots$ & -2.9 & $<81$ & $<1.55 \times 10^{12}$ \\
\hline $1016347+275149$ & LHSa2243 & M8.0 & 14 & $\begin{array}{l}\cdots \\
<3\end{array}$ & -3.38 & -3.87 & $\ldots$ & $<84$ & $<1.97 \times 10^{13}$ \\
\hline $1024099+181553$ & 2MUCDa10906 & M8.0 & 16 & 5 & -3.38 & -4.84 & $\begin{array}{l}\cdots \\
\cdots\end{array}$ & $<87$ & $<2.67 \times 10^{13}$ \\
\hline $1309218-233035$ & & M8.0 & 16 & 7 & -3.63 & -4.35 & $\begin{array}{l}\cdots \\
\cdots\end{array}$ & $<93$ & $<2.85 \times 10^{13}$ \\
\hline $1428041+135613$ & LHS 2919 & M8.0 & 10 & $\ldots$ & -3.37 & $\ldots$ & $\begin{array}{l}\cdots \\
\cdots\end{array}$ & $<90$ & $<1.08 \times 10^{13}$ \\
\hline $1440229+133923$ & & M8.0 & 18 & $\begin{array}{l}\cdots \\
<3\end{array}$ & -3.33 & -4.60 & $\begin{array}{l}\cdots \\
\cdots\end{array}$ & $<75$ & $<2.91 \times 10^{13}$ \\
\hline $1444171+300214$ & LP 326-21 & M8.0 & 13 & $\ldots$ & -3.61 & $\ldots$ & $\begin{array}{l}\cdots \\
\cdots\end{array}$ & $<81$ & $<1.64 \times 10^{13}$ \\
\hline 2206227-204706 & & M8.0 & 27 & 24 & -2.95 & -4.54 & $\begin{array}{l}\cdots \\
\cdots\end{array}$ & $<84$ & $<7.33 \times 10^{13}$ \\
\hline $2349489+122438$ & LP 523-55 & M8.0 & 20 & 4 & -3.31 & -4.61 & $\begin{array}{l}\cdots \\
\cdots\end{array}$ & $<60$ & $<2.87 \times 10^{13}$ \\
\hline 2351504-253736A & & M8.0 & 18 & 36 & -3.36 & -4.61 & $\begin{array}{l}\cdots \\
\cdots\end{array}$ & $<69$ & $<2.67 \times 10^{13}$ \\
\hline $18261131+3014201$ & LSR J1826+3014 & M8.5 & 13.9 & $\ldots$ & $\ldots$ & $\ldots$ & $\begin{array}{l}\cdots \\
\cdots\end{array}$ & $<87$ & $<2.01 \times 10^{13}$ \\
\hline $0335020+234235$ & & M8.5 & 19 & $\begin{array}{l}\cdots \\
\cdots\end{array}$ & -3.61 & $\begin{array}{l}\cdots \\
\cdots\end{array}$ & $\begin{array}{l}\cdots \\
\cdots\end{array}$ & $<69$ & $<2.98 \times 10^{13}$ \\
\hline $1454290+160605$ & GJ 569Ba & M8.5 & 10 & $\begin{array}{l}\cdots \\
\cdots\end{array}$ & -3.80 & $\begin{array}{l}\cdots \\
\cdots\end{array}$ & -4.3 & $<30$ & $<3.59 \times 10^{12}$ \\
\hline $1501081+225002$ & TVLM513-465 & M8.5 & 11 & 60 & -3.59 & $\cdots$ & -5.1 & $\begin{array}{c}190 \pm 15 \\
980 \pm 40 \\
4000\end{array}$ & $\begin{array}{l}2.75 \times 10^{13} \\
1.42 \times 10^{14} \\
5.79 \times 10^{14}\end{array}$ \\
\hline $1835379+325954$ & LSR J1835+3 & M8.5 & 6 & 44 & -3.93 & -4.85 & $<-5.6$ & $525 \pm 15$ & $2.26 \times 10^{13}$ \\
\hline $0140026+270150$ & & M8.5 & 19 & 6.5 & -3.32 & $\ldots$ & $\ldots$ & $<20$ & $<8.64 \times 10^{12}$ \\
\hline $1121492-131308$ & GJ 3655 & M8.5 & 12 & 27 & -3.68 & -3.87 & $\begin{array}{l}\cdots \\
\cdots\end{array}$ & $<102$ & $<1.76 \times 10^{13}$ \\
\hline $1124048+380805$ & & M8.5 & 19 & 7.5 & -3.41 & -5.16 & $\begin{array}{l}\cdots \\
\cdots\end{array}$ & $<66$ & $<2.85 \times 10^{13}$ \\
\hline $1403223+300754$ & & M8.5 & 19 & 10 & -3.39 & -4.49 & $\begin{array}{l}\cdots \\
\cdots\end{array}$ & $<60$ & $<2.59 \times 10^{13}$ \\
\hline $2353594-083331$ & & M8.5 & 22 & 4.5 & -3.41 & -4.42 & $\begin{array}{l}\cdots \\
\cdots\end{array}$ & $<69$ & $<3.99 \times 10^{13}$ \\
\hline $14284323+3310391$ & GJ 3849 & M9 & 11 & 10 & -3.62 & -4.7 & $<-4.35$ & $<63$ & $<9.12 \times 10^{12}$ \\
\hline $0019457+521317$ & & M9.0 & 19 & 9 & -3.95 & -4.29 & $\ldots$ & $<42$ & $<1.81 \times 10^{13}$ \\
\hline $0109511-034326$ & LP 647-13 & M9.0 & 11 & 13 & -3.98 & -4.50 & $\ldots$ & $<33$ & $<4.78 \times 10^{12}$ \\
\hline $0339352-352544$ & LP 944-20 & M9.0 & 5 & 26 & -3.79 & -5.30 & $\begin{array}{c}<-6.3 \\
-3.7(f)\end{array}$ & $\begin{array}{c}74 \pm 13 \\
2600+200\end{array}$ & $\begin{array}{l}2.21 \times 10^{12} \\
7.78 \times 10^{13}\end{array}$ \\
\hline $0434152+225031$ & & M9.0 & 140 & $\ldots$ & -2.53 & $\ldots$ & & $<69$ & $<1.62 \times 10^{15}$ \\
\hline $0436389+225812$ & & M9.0 & 140 & $\ldots$ & -2.59 & $\cdots$ & -3.7 & $<57$ & $<1.34 \times 10^{15}$ \\
\hline 0537259-023432 & & M9.0 & 352 & $\ldots$ & -3.56 & $\cdots$ & $\ldots$ & $<66$ & $<9.78 \times 10^{15}$ \\
\hline $0810586+142039$ & & M9.0 & 20 & $\dddot{11}$ & -3.39 & -4.17 & $\begin{array}{l}\cdots \\
\ldots\end{array}$ & $<39$ & $<1.87 \times 10^{13}$ \\
\hline $0853362-032932$ & GJ 3517 & M9.0 & 9 & 13.5 & -3.49 & -3.93 & $\begin{array}{c}-3.7 \\
-2.5(\mathrm{f})\end{array}$ & $<81$ & $<7.85 \times 10^{12}$ \\
\hline $1454280+160605$ & GJ 569Bb & M9.0 & 10 & $\ldots$ & -4.04 & $\ldots$ & -2.6 & $<30$ & $<3.59 \times 10^{12}$ \\
\hline $1627279+810507$ & & M9.0 & 21 & $\cdots$ & -3.45 & $\cdots$ & $\ldots$ & $<60$ & $<3.17 \times 10^{13}$ \\
\hline $1707183+643933$ & & M9.0 & 17 & $\cdots$ & -3.44 & $\cdots$ & $\begin{array}{l}\cdots \\
\cdots\end{array}$ & $<60$ & $<2.07 \times 10^{13}$ \\
\hline $0443376+000205$ & & M9.0 & 16 & 13.5 & -3.47 & -5.00 & $\begin{array}{l}\cdots \\
\ldots\end{array}$ & $<54$ & $<1.65 \times 10^{13}$ \\
\hline $1224522-123835$ & & M9.0 & 17 & $\begin{array}{c}15.5 \\
7\end{array}$ & $\begin{array}{l}-3.41 \\
-3.94\end{array}$ & -4.52 & $\begin{array}{l}\cdots \\
\ldots\end{array}$ & $<102$ & $<3.53 \times 10^{13}$ \\
\hline $1411213-211950$ & & M9.0 & 16 & 44 & -3.93 & -4.93 & $\begin{array}{l}\cdots \\
\ldots\end{array}$ & $<93$ & $<2.85 \times 10^{13}$ \\
\hline $1428432+331039$ & LHS 2924 & M9.0 & 11 & $\begin{array}{l}44 \\
11\end{array}$ & $\begin{array}{l}-5.95 \\
-3.59\end{array}$ & $\begin{array}{l}-4.93 \\
-5.14\end{array}$ & $<-4.35$ & $<84$ & $<1.22 \times 10^{13}$ \\
\hline $1707234-055824$ & 2MUCD 20701 & M9.0 & 15 & $\ldots$ & -3.31 & $\ldots$ & $\ldots$ & $\begin{array}{l}<81 \\
<48\end{array}$ & $\begin{array}{l}<2.80 \times 10^{13} \\
<129 \times 10^{13}\end{array}$ \\
\hline $2200020-303832 \mathrm{AB}$ & & M9.0 & 35 & 17 & -3.17 & -5.03 & $\ldots$ & $<78$ & $<1.14 \times 10^{14}$ \\
\hline $0024246-015819$ & BRI B0021-0 & M9.5 & 12 & 33 & $\begin{array}{l}-3.50 \\
-3.45\end{array}$ & $\begin{array}{l}-6.12 \\
-6.12\end{array}$ & $<-5.0$ & $\begin{array}{c}83 \pm 18 \\
<60\end{array}$ & $\begin{array}{c}1.43 \times 10^{13} \\
<1.03 \times 10^{13}\end{array}$ \\
\hline $0027420+050341$ & PC $0025+044$ & M9.5 & 72 & 13 & -3.62 & -3.39 & $<-3.8$ & $<75$ & $<4.65 \times 10^{14}$ \\
\hline $0109217+294925$ & & M9.5 & 19 & 7 & -3.49 & $\ldots$ & $\ldots$ & $<54$ & $<2.33 \times 10^{13}$ \\
\hline $0149089+295613$ & & M9.5 & 17 & 12 & -3.74 & $\begin{array}{l}\cdots \\
\cdots\end{array}$ & $\begin{array}{l}\cdots \\
\cdots\end{array}$ & $<140$ & $<4.84 \times 10^{13}$ \\
\hline $1438082+640836$ & & M9.5 & 18 & 12 & -4.08 & -4.77 & $\begin{array}{l}\cdots \\
\cdots\end{array}$ & $<105$ & $<4.07 \times 10^{13}$ \\
\hline $2237325+392239$ & G216-7B & M9.5 & 19 & $\ldots$ & -3.66 & -5.02 & $\begin{array}{l}\cdots \\
\cdots\end{array}$ & $<81$ & $<3.50 \times 10^{13}$ \\
\hline $17312974+2721233$ & LSPM J1731+2721 & L0 & 11.8 & 15 & -3.74 & -4.6 & $\ldots$ & $<57$ & $<9.50 \times 10^{12}$ \\
\hline $1421314+182740$ & & L0.0 & 20 & $\ldots$ & -3.56 & $\ldots$ & $\cdots$ & $<42$ & $<2.01 \times 10^{13}$ \\
\hline $0345431+254023$ & & L0.0 & 27 & $\ldots$ & -3.56 & $\ldots$ & $\ldots$ & $<87$ & $<7.59 \times 10^{13}$ \\
\hline $0314034+160305$ & & L0.0 & 14 & 19 & -3.59 & -4.69 & $\begin{array}{l}\cdots \\
\cdots\end{array}$ & $<108$ & $<2.53 \times 10^{13}$ \\
\hline $1159385+005726$ & & L0.0 & 30 & 71 & -3.57 & -5.06 & $\begin{array}{l}\cdots \\
\cdots\end{array}$ & $<54$ & $<5.81 \times 10^{13}$ \\
\hline $1221277+025719$ & & L0.0 & 19 & 25 & -3.59 & -4.88 & $\ldots$ & $<78$ & $<3.37 \times 10^{13}$ \\
\hline $1731297+272123$ & & L0.0 & 12 & 15 & -3.56 & -4.80 & $\ldots$ & $<69$ & $<1.19 \times 10^{13}$ \\
\hline $1854459+842947$ & & L0.0 & 23 & 7 & -3.62 & -4.73 & $\ldots$ & $<87$ & $<5.51 \times 10^{13}$ \\
\hline $0746425+200032$ & & L0.5 & 12 & 31 & -3.93 & -5.29 & $<-4.7$ & $\begin{array}{c}224 \pm 15 \\
15000 \pm 100\end{array}$ & $\begin{array}{l}3.86 \times 10^{13} \\
2.58 \times 10^{15}\end{array}$ \\
\hline $1412244+163311$ & & L0.5 & 25 & 19 & -3.61 & -5.50 & $\ldots$ & $<69$ & $<5.16 \times 10^{13}$ \\
\hline $1441371-094559$ & & L0.5 & 28 & 23 & -3.59 & -5.48 & $\ldots$ & $<84$ & $<7.88 \times 10^{13}$ \\
\hline
\end{tabular}


Table A.1. continued.

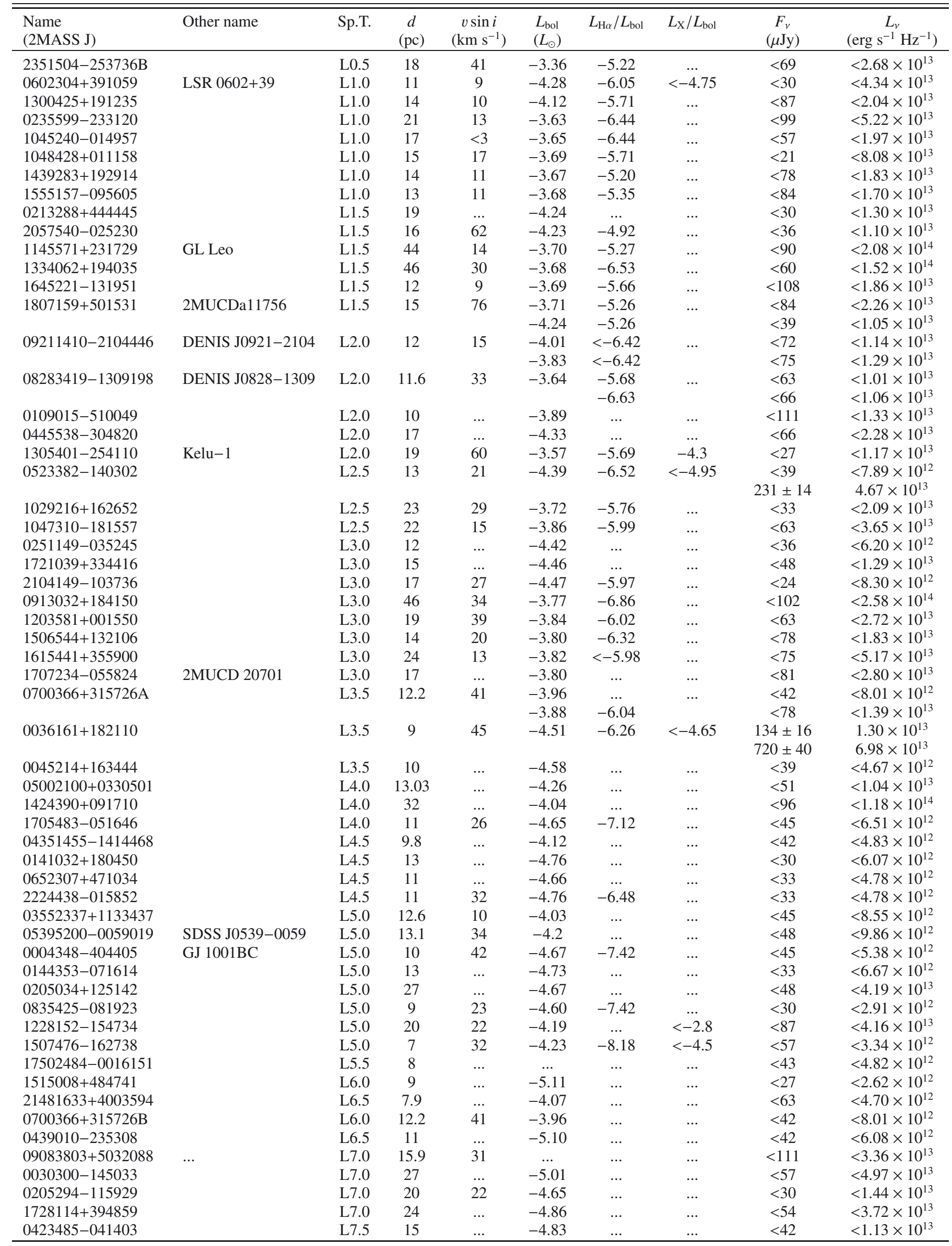


Table A.1. continued.

\begin{tabular}{|c|c|c|c|c|c|c|c|c|c|}
\hline $\begin{array}{l}\text { Name } \\
(2 \mathrm{MASS} J)\end{array}$ & Other name & Sp.T. & $\begin{array}{c}d \\
(\mathrm{pc})\end{array}$ & $\begin{array}{c}v \sin i \\
\left(\mathrm{~km} \mathrm{~s}^{-1}\right)\end{array}$ & $\begin{array}{l}L_{\text {bol }} \\
\left(L_{\odot}\right)\end{array}$ & $L_{\mathrm{H} \alpha} / L_{\mathrm{bol}}$ & $L_{\mathrm{X}} / L_{\text {bol }}$ & $\begin{array}{c}F_{v} \\
(\mu \mathrm{Jy})\end{array}$ & $\begin{array}{c}L_{V} \\
\left(\mathrm{erg} \mathrm{s}^{-1} \mathrm{~Hz}^{-1}\right)\end{array}$ \\
\hline $0825196+211552$ & & L7.5 & 11 & 19 & -5.21 & -8.18 & $\ldots$ & $<45$ & $<6.51 \times 10^{12}$ \\
\hline $2252107-173013$ & & L7.5 & 8 & $\ldots$ & -5.29 & $\ldots$ & $\begin{array}{l}\cdots \\
\cdots\end{array}$ & $<30$ & $<2.30 \times 10^{12}$ \\
\hline $08575849+5708514$ & SDSS J0857+5708 & L 8.0 & 11 & $\begin{array}{l}\cdots \\
\ldots\end{array}$ & $\ldots$ & $\begin{array}{l}\cdots \\
\cdots\end{array}$ & $\begin{array}{l}\cdots \\
\cdots\end{array}$ & $<51$ & $<7.38 \times 10^{12}$ \\
\hline $02572581-3105523$ & DENIS J0257-3105 & L8.0 & 9.6 & $\begin{array}{l}\cdots \\
\ldots\end{array}$ & -4.82 & $\cdots$ & $\begin{array}{l}\cdots \\
\ldots\end{array}$ & $<93$ & $<1.03 \times 10^{13}$ \\
\hline $0929336+342952$ & & L 8.0 & 22 & $\begin{array}{l}\cdots \\
\cdots\end{array}$ & -5.25 & $\begin{array}{l}\cdots \\
\cdots\end{array}$ & $\begin{array}{l}\cdots \\
\cdots\end{array}$ & $<42$ & $<2.43 \times 10^{13}$ \\
\hline $1523226+301456$ & & L8.0 & 19 & $\begin{array}{l}\cdots \\
\ldots\end{array}$ & -5.27 & $\cdots$ & $\begin{array}{l}\cdots \\
\ldots\end{array}$ & $<45$ & $<1.93 \times 10^{13}$ \\
\hline $1632291+190441$ & & L8.0 & 15 & 30 & -5.31 & $\cdots$ & $\begin{array}{l}\cdots \\
\ldots\end{array}$ & $<54$ & $<1.45 \times 10^{13}$ \\
\hline $08300825+4828482$ & SDSS J0830+4828 & L9.0 & 13 & $\ldots$ & -4.58 & $\ldots$ & $\ldots$ & $<87$ & $<1.78 \times 10^{13}$ \\
\hline $0151415+124430$ & & T0.0 & 21 & $\ldots$ & -5.37 & $\ldots$ & $\ldots$ & $<51$ & $<2.69 \times 10^{13}$ \\
\hline 2204105-564657A & & $\mathrm{T} 1.0$ & 4 & $\begin{array}{l}\cdots \\
\ldots\end{array}$ & -5.03 & $\cdots$ & $\begin{array}{l}\cdots \\
\ldots\end{array}$ & $<72$ & $<1.38 \times 10^{12}$ \\
\hline $01365662+0933473$ & SIMP J0136+0933 & $\mathrm{T} 2.5$ & $\begin{array}{c}6.4 \\
8.27\end{array}$ & $\begin{array}{l}\cdots \\
\cdots\end{array}$ & $\ldots$ & $\begin{array}{l}\cdots \\
\cdots\end{array}$ & $\begin{array}{l}\cdots \\
\cdots\end{array}$ & $<75$ & $\begin{array}{l}<3.68 \times 10^{13} \\
<6.14 \times 10^{13}\end{array}$ \\
\hline $0207428+000056$ & & $\mathrm{~T} 4.0$ & $\begin{array}{l}0.21 \\
29\end{array}$ & $\ldots$ & -5.21 & $\ldots$ & $\ldots$ & $<39$ & $<3.92 \times 10^{13}$ \\
\hline $0559191-140448$ & & $\mathrm{~T} 4.5$ & 10 & $\begin{array}{l}\cdots \\
\ldots\end{array}$ & -4.53 & $<-6.1$ & $\begin{array}{l}\cdots \\
\cdots\end{array}$ & $<27$ & $<3.23 \times 10^{12}$ \\
\hline $15031961+2525196$ & & $\mathrm{~T} 5.5$ & 7 & 36 & $\ldots$ & $<-5.5$ & $\begin{array}{l}\cdots \\
\cdots\end{array}$ & $<90$ & $<5.28 \times 10^{12}$ \\
\hline $1534498-295227$ & & T5.5 & 14 & ... & -5.00 & $<-5.2$ & $\begin{array}{l}\cdots \\
\cdots\end{array}$ & $<63$ & $<1.48 \times 10^{13}$ \\
\hline $09373487+2931409$ & & T6.0 & 6.14 & $\begin{array}{l}\cdots \\
\ldots\end{array}$ & -5.28 & $<-6.0$ & $\begin{array}{l}\cdots \\
\ldots\end{array}$ & $<66$ & $<2.98 \times 10^{12}$ \\
\hline $21543318+5942187$ & & T6.0 & $\begin{array}{l}0.14 \\
18.8\end{array}$ & $\begin{array}{l}\cdots \\
\ldots\end{array}$ & $\ldots$ & $\ldots$ & $\begin{array}{l}\cdots \\
\ldots\end{array}$ & $<60$ & $<2.54 \times 10^{13}$ \\
\hline $1624144+002916$ & & T6.0 & $\begin{array}{c}10.0 \\
11\end{array}$ & $\begin{array}{l}\cdots \\
\ldots\end{array}$ & -5.16 & $\begin{array}{l}\cdots \\
\cdots\end{array}$ & $\begin{array}{l}\cdots \\
\ldots\end{array}$ & $<36$ & $<5.21 \times 10^{12}$ \\
\hline $2204105-564657 \mathrm{~B}$ & & T6.0 & $\begin{array}{c}11 \\
4\end{array}$ & $\begin{array}{l}\cdots \\
\ldots\end{array}$ & -5.03 & $\begin{array}{l}\cdots \\
\ldots\end{array}$ & $\begin{array}{l}\cdots \\
\ldots\end{array}$ & $<72$ & $<1.34 \times 10^{12}$ \\
\hline $1047539+212423$ & & $\mathrm{~T} 6.5$ & 11 & $\begin{array}{l}\cdots \\
\cdots\end{array}$ & -5.35 & -5.4 & $\begin{array}{l}\cdots \\
\cdots\end{array}$ & $\begin{array}{l}<45 \\
\sim 1500\end{array}$ & $\begin{array}{l}<6.51 \times 10^{12} \\
\sim 2.17 \times 10^{14}\end{array}$ \\
\hline $1346464-003150$ & & T6.5 & 15 & $\ldots$ & -5.00 & $\ldots$ & $\ldots$ & $\begin{array}{l}\sim 1500 \\
<105\end{array}$ & $<2.83 \times 10^{13}$ \\
\hline $07271824+1710012$ & & $\mathrm{~T} 7.0$ & 9.09 & $\cdots$ & -5.26 & $<-5.7$ & $\begin{array}{l}\cdots \\
\cdots\end{array}$ & $<54$ & $<5.34 \times 10^{12}$ \\
\hline $0610351-215117$ & GJ 229B & $\mathrm{T} 7.0$ & 6 & $\begin{array}{l}\cdots \\
\cdots\end{array}$ & -5.21 & $\ldots$ & $<-1.26$ & $<69$ & $<2.97 \times 10^{12}$ \\
\hline $11145133-2618235$ & & $\mathrm{~T} 7.5$ & $\begin{array}{c}7 \\
10\end{array}$ & $\begin{array}{l}\cdots \\
\ldots\end{array}$ & $\ldots$ & $\begin{array}{l}\cdots \\
\cdots\end{array}$ & $\ldots$ & $<60$ & $\begin{array}{l}<3.52 \times 10^{12} \\
<7.18 \times 10^{12}\end{array}$ \\
\hline $1217111-031113$ & & $\mathrm{~T} 7.5$ & 11 & $\ldots$ & -5.32 & $<-5.3$ & $\ldots$ & $<111$ & $<1.61 \times 10^{13}$ \\
\hline $09393548-2448279$ & & $\mathrm{~T} 8.0$ & $\begin{array}{c}8.7 \\
5.34\end{array}$ & $\cdots$ & -5.69 & $\ldots$ & $\cdots$ & $<54$ & $\begin{array}{l}<4.89 \times 10^{12} \\
<184 \times 10^{12}\end{array}$ \\
\hline $14571496-2121477$ & GL570D & $\mathrm{T} 8.0$ & 5.88 & 30 & -5.52 & $<-5.3$ & $<-1.85$ & $<54$ & $<2.23 \times 10^{12}$ \\
\hline 0415195-093506 & & $\mathrm{T} 8.0$ & 6 & $\ldots$ & -5.73 & $<-5.4$ & $\ldots$ & $<45$ & $<1.94 \times 10^{12}$ \\
\hline
\end{tabular}

